\title{
Direct Derivation of the Comma 3-Vertex in the Full String Basis
}

\author{
A. Abdurrahman', M. Gassem² \\ ${ }^{1}$ Department of Physics, Shippensburg University of Pennsylvania, Shippensburg, PA, USA \\ ${ }^{2}$ The Division of Mathematics and Science, South Texas College, McAllen, TX, USA \\ Email: Ababdu@ship.edu,mgassem@southtexascollege.edu
}

How to cite this paper: Abdurrahman, A. and Gassem, M. (2019) Direct Derivation of the Comma 3-Vertex in the Full String Basis. Journal of Modern Physics, 10, 1271-1298.

https://doi.org/10.4236/jmp.2019.1010085

Received: August 18, 2019

Accepted: September 26, 2019

Published: September 29, 2019

Copyright $\odot 2019$ by author(s) and Scientific Research Publishing Inc. This work is licensed under the Creative Commons Attribution International License (CC BY 4.0).

http://creativecommons.org/licenses/by/4.0/

\begin{abstract}
The interacting comma 3-vertex for the bosonic open string in the full string basis is derived using the half string overlap relations directly. Thus avoiding the coherent states technique employed in earlier derivations. The resulting form of the interacting 3-vertex turns out to be precisely the desired expression obtained in terms of the full string oscillator modes. This derivation establishes that the comma 3-vertex and Witten's 3-vertex are identical and therefore are interchangeable.
\end{abstract}

\section{Keywords}

String Theory, Witten's Theory, Open Bosonic String

\section{Introduction}

Here we are going to give a brief derivation of the transformation matrices between the half string coordinates and the full string coordinates needed for the construction of the half string interacting vertex in terms of the oscillator representation of the full string. For this we shall follow closely the discussion of reference [1] [2] [3] [4] [5]. To make this more concrete, we recall the standard mode expansion for the open bosonic string coordinate

$$
x^{\mu}(\sigma)=x_{0}^{\mu}+\sqrt{2} \sum_{n=1}^{\infty} x_{n}^{\mu} \cos (n \sigma), \quad \sigma \in[0, \pi]
$$

where $\mu=1,2, \cdots, 27$ and $x^{27}(\sigma)$ correspond to the ghost part $\phi(\sigma)$. The half string coordinates $x^{L, \mu}(\sigma)$ and $x^{R, \mu}(\sigma)$ for the left and right halves of the string are defined in the usual way

$$
\begin{aligned}
& x^{L, \mu}(\sigma)=x^{\mu}(\sigma)-x^{\mu}\left(\frac{\pi}{2}\right), \quad \sigma \in\left[0, \frac{\pi}{2}\right] \\
& x^{R, \mu}(\sigma)=x^{\mu}(\pi-\sigma)-x^{\mu}\left(\frac{\pi}{2}\right), \quad \sigma \in\left[0, \frac{\pi}{2}\right]
\end{aligned}
$$


where both $x^{L, \mu}(\sigma)$ and $x^{R, \mu}(\sigma)$ satisfy the usual Neumann boundary conditions at $\sigma=0$ and a Dirichlet boundary conditions $\sigma=\pi / 2$. Thus they have expansions of the form

$$
\begin{aligned}
& x^{L, \mu}(\sigma)=\sqrt{2} \sum_{n=1}^{\infty} x_{n}^{L \mu} \cos (n \sigma), \\
& x^{R, \mu}(\sigma)=\sqrt{2} \sum_{n=1}^{\infty} x_{n}^{R \mu} \cos (n \sigma)
\end{aligned}
$$

Comparing Equation (1) and Equation (3) we obtain an expression for the half string modes in terms of the full string modes

$$
\begin{aligned}
& x_{n}^{L, \mu}=x_{2 n-1}^{\mu}+\sum_{m=1}^{\infty} \sqrt{\frac{2 m}{2 n-1}}\left[M_{m n}^{1}+M_{m n}^{2}\right] x_{2 m}^{\mu}, \\
& x_{n}^{R, \mu}=-x_{2 n-1}^{\mu}+\sum_{m=1}^{\infty} \sqrt{\frac{2 m}{2 n-1}}\left[M_{m n}^{1}+M_{m n}^{2}\right] x_{2 m}^{\mu}
\end{aligned}
$$

where the change of representation matrices are given by

$$
\begin{aligned}
& M_{m n}^{1}=\frac{2}{\pi} \sqrt{\frac{2 m}{2 n-1}} \frac{(-1)^{m+n}}{2 m-(2 n-1)}, m, n=1,2,3, \cdots \\
& M_{m n}^{2}=\frac{2}{\pi} \sqrt{\frac{2 m}{2 n-1}} \frac{(-1)^{m+n}}{2 m+(2 n-1)}, m, n=1,2,3, \cdots
\end{aligned}
$$

Since the transformation in (4) is non singular, one may invert the relation in (4). Inverting (4) we find

$$
\begin{aligned}
& x_{2 n-1}^{\mu}=\frac{1}{2}\left(x_{n}^{L, \mu}-x_{n}^{R, \mu}\right), \\
& x_{2 n}^{\mu}=\frac{1}{2} \sum_{m=1}^{\infty} \sqrt{\frac{2 m-1}{2 n}}\left[M_{m n}^{1}-M_{m n}^{2}\right]\left(x_{m}^{L, \mu}+x_{m}^{R, \mu}\right)
\end{aligned}
$$

where $n=1,2,3, \cdots$.

In the decomposition of the string into right and left pieces in (2), we singled out the midpoint coordinate. Consequently the relationship between $x_{n}^{\mu}$ and $\left(x_{n}^{L, \mu}, x_{n}^{R, \mu}\right)$ does not involve the zero mode $x_{0}^{\mu}$ of $x^{\mu}(\sigma)$. At $\sigma=\pi / 2$, we have

$$
x_{M}^{\mu} \equiv x^{\mu}\left(\frac{\pi}{2}\right)=x_{0}^{\mu}+\sqrt{2} \sum_{n=1}^{\infty} x_{2 n}^{\mu}
$$

and so the center of mass $x_{0}^{\mu}$ may be related to the half string coordinates and the midpoint coordinate

$$
x_{0}^{\mu}=x_{M}^{\mu}-\frac{\sqrt{2}}{\pi} \sum_{n=1}^{\infty} \frac{(-1)^{n}}{2 n-1}\left(x_{n}^{L, \mu}+x_{n}^{R, \mu}\right)
$$

Equations (8) and (9) with Equations (4) and (7) complete the equivalence between $x_{n}^{\mu}, n=0,1,2, \cdots$, and $\left(x_{n}^{L, \mu}, x_{n}^{R, \mu}, x_{M}^{\mu}\right), n=1,2,3, \cdots$.

For later use we also need the relationships between $\left\{p_{n}^{L, \mu}, p_{n}^{R, \mu}, p_{M}^{\mu}\right\}_{n=1}^{\infty}$, the half string conjugate momenta and $\left\{p_{n}^{\mu}\right\}_{n=0}^{\infty}$, the full string conjugate momenta. Using Dirac quantization procedure 


$$
\left[x_{n}^{r}, p_{m}^{s}\right]=i \delta^{r s} \delta_{n m},
$$

we find (thereafter; the space-time index $\mu$ is suppressed),

$$
\begin{aligned}
& p_{n}^{L}=\frac{1}{2} p_{2 n-1}+\sum_{m=1}^{\infty} \sqrt{\frac{2 n-1}{2 m}}\left[M_{m n}^{1}-M_{m n}^{2}\right] p_{2 m}-\frac{\sqrt{2}}{\pi} \frac{(-1)^{n}}{2 n-1} p_{0}, \\
& p_{n}^{R}=-\frac{1}{2} p_{2 n-1}+\sum_{m=1}^{\infty} \sqrt{\frac{2 n-1}{2 m}}\left[M_{m n}^{1}-M_{m n}^{2}\right] p_{2 m}-\frac{\sqrt{2}}{\pi} \frac{(-1)^{n}}{2 n-1} p_{0}
\end{aligned}
$$

and

$$
p_{M}=p_{0}
$$

To obtain the full string conjugate momenta in terms of the half string conjugate momenta, we need to invert the above relations; skipping the technical details we find

$$
\begin{aligned}
& p_{2 n-1}=p_{n}^{L}-p_{n}^{R}, \\
& p_{2 n}=\sum_{m=1}^{\infty} \sqrt{\frac{2 n}{2 m-1}}\left[M_{n m}^{1}+M_{n m}^{2}\right]\left(p_{m}^{L}+p_{m}^{R}\right)+\sqrt{2}(-1)^{n} p_{M}
\end{aligned}
$$

We notice that the existence of the one-to-one correspondence between the half string and the full string degrees of freedom guarantees the existence of the identification

$$
\bar{H}=\overline{H_{M} \otimes H_{L} \otimes H_{R}}
$$

where $\bar{H}$ stands for the completion of the full string Hilbert space and $H_{L}$, $H_{R}, H_{M}$ in the tensor product stand for the two half-string Hilbert spaces and the Hilbert space of functions of the mid-point, respectively.

\section{The Half-String Overlaps}

The half string three interaction vertex of the open bosonic string $\left(V_{x}^{H S}\right)$ have been constructed in the half-string oscillator representation [2] [3]. Here we are interested in constructing the comma three interaction vertex in terms of the oscillator representation of the full string. Here we shall only consider the coordinate piece of the comma three interaction vertex. The ghost part of the vertex $\left(V_{\phi}^{H S}\right)$ in the bosonic representation is identical to the coordinate piece apart from the ghost mid-point insertions $3 i \phi(\pi / 2) / 2$ required for ghost number conservation at the mid-point. To simplify the calculation we introduce a new set of coordinates and momenta based on a $Z_{3}$ Fourier transform ${ }^{1}$

$$
\left(\begin{array}{c}
Q^{r}(\sigma) \\
\bar{Q}^{r}(\sigma) \\
Q^{3, r}(\sigma)
\end{array}\right)=\frac{1}{\sqrt{3}}\left(\begin{array}{ccc}
e & \bar{e} & 1 \\
\bar{e} & e & 1 \\
1 & 1 & 1
\end{array}\right)\left(\begin{array}{c}
\chi^{1, r}(\sigma) \\
\chi^{2, r}(\sigma) \\
\chi^{3, r}(\sigma)
\end{array}\right)
$$

where $e=\exp (2 \pi i / 3)$ and $r$ refers to the left $(L)$ and right $(R)$ parts of the string. The superscripts 1,2 and 3 refers to string 1 , string 2 and string 3 , respectively. Similarly one obtains a new set for the conjugate momenta $\wp^{r}(\sigma)$,

${ }^{1}$ This technique was first used by D. Gross and A. Jevicki in 1986. 
$\bar{\wp}^{r}(\sigma)$ and $\wp^{3, r}(\sigma)$ as well as a new set for the creation-annihilation operators $\left(B_{j}^{r}, B_{j}^{r \dagger}\right)$. In the $Z_{3}$ Fourier space the degrees of freedom in the $\delta$ function overlaps equations decouple which result in a considerable reduction of the amount of algebra involved in such calculations as we shall see shortly. Notice that in the $Z_{3}$ Fourier space the commutation relations are

$$
\begin{gathered}
{\left[Q^{r}(\sigma), \bar{\wp}^{s}\left(\sigma^{\prime}\right)\right]=i \delta^{r s} \delta\left(\sigma-\sigma^{\prime}\right)} \\
{\left[\bar{Q}^{r}(\sigma), \wp^{s}\left(\sigma^{\prime}\right)\right]=i \delta^{r s} \delta\left(\sigma-\sigma^{\prime}\right)} \\
{\left[Q^{3, r}(\sigma), \wp^{3, s}\left(\sigma^{\prime}\right)\right]=i \delta^{r s} \delta\left(\sigma-\sigma^{\prime}\right)}
\end{gathered}
$$

Since $\left[Q^{r}(\sigma), \wp^{s}\left(\sigma^{\prime}\right)\right] \neq i \delta^{r s} \delta\left(\sigma-\sigma^{\prime}\right)$, then $Q^{r}(\sigma)$ and $\wp^{r}(\sigma)$ are no longer canonical variables. The canonical variables in this case are $Q^{r}(\sigma)$ and $\bar{\wp}^{r}(\sigma)$. Thus the $Z_{3}$ Fourier transform does not conserve the original commutation relations. The variables $Q^{3, r}(\sigma)$ and $\wp^{3, s}(\sigma)$ are still canonical however. This is a small price to pay for decoupling string three in the $Z_{3}$ Fourier space from the other two strings as we shall see in the construction of the comma three interaction vertex. Recall that the overlap equations for the comma three interacting vertex are given by

$$
\begin{gathered}
\chi^{j, r}(\sigma)=\chi^{j-1, r-1}(\sigma), \quad 0 \leq \sigma \leq \pi / 2 \\
x_{M}^{1}=x_{M}^{2}=x_{M}^{3}
\end{gathered}
$$

for the coordinates (where the mid-point coordinate $x_{M} \equiv x(\pi / 2)$ and the identifications $j-1=0 \equiv 3$ and $r-1=0 \equiv R$ are understood). The comma coordinates are defined in the usual way [1]

$$
\begin{array}{cc}
\chi^{j, L}(\sigma)=x(\sigma)-x\left(\frac{\pi}{2}\right), & 0 \leq \sigma \leq \pi / 2 \\
\chi^{j, R}(\sigma)=x(\pi-\sigma)-x\left(\frac{\pi}{2}\right), & 0 \leq \sigma \leq \pi / 2
\end{array}
$$

The overlaps for the canonical momenta are given by

$$
\begin{gathered}
\wp^{j, r}(\sigma)=-\wp^{j-1, r-1}(\sigma), \quad 0 \leq \sigma \leq \pi / 2 \\
\wp_{M}^{1}+\wp_{M}^{2}+\wp_{M}^{3}=0
\end{gathered}
$$

where the mid-point momentum is defined in the usual way $\wp_{M} \equiv-i \partial / \partial x_{M}=-i \partial / \partial x_{0}=p_{0}$. The comma coordinates and their canonical momenta obey the usual commutation relations

$$
\left[\chi^{j, r}(\sigma), \wp^{j, s}\left(\sigma^{\prime}\right)\right]=i \delta^{r s} \delta\left(\sigma-\sigma^{\prime}\right), r, s=L, R
$$

In $Z_{3}$ Fourier space of the comma, the overlap equations for the half string coordinates read

$$
\begin{gathered}
Q^{L}(\sigma)=e Q^{R}(\sigma), \quad 0 \leq \sigma \leq \pi / 2 \\
\bar{Q}^{L}(\sigma)=\bar{e} \bar{Q}^{R}(\sigma), \quad 0 \leq \sigma \leq \pi / 2 \\
Q_{M}=\bar{Q}_{M}=0
\end{gathered}
$$




$$
\begin{gathered}
Q^{3, L}(\sigma)=Q^{3, R}(\sigma), \quad 0 \leq \sigma \leq \pi / 2 \\
Q_{M}^{3}=Q_{M}^{3}
\end{gathered}
$$

where Equation (29) is to be understood as an overlap Equation (i.e., its action on the three vertex is zero). Similarly the canonical momenta of the half string in the $Z_{3}$ Fourier space of the comma translate into

$$
\begin{array}{cc}
\wp^{L}(\sigma)=-e \wp^{R}(\sigma), & 0 \leq \sigma \leq \pi / 2 \\
\wp^{L}(\sigma)=-\bar{e} \wp^{R}(\sigma), & 0 \leq \sigma \leq \pi / 2 \\
\wp^{3, L}(\sigma)=-\wp^{3, R}(\sigma), \quad 0 \leq \sigma \leq \pi / 2 \\
P_{M}^{3}=0
\end{array}
$$

The overlap conditions on $Q^{r}(\sigma)$ and $\wp^{r}(\sigma)$ determine the form of the comma three interaction vertex. Thus in the $Z_{3}$ Fourier space of the comma the overlap equations separate into two sets. The half string three vertex

$$
V_{x}^{H S}\left(b^{1, r \uparrow}, b^{2, r \uparrow}, b^{3, r \uparrow}\right)
$$

therefore separates into a product of two pieces one depending on $B^{3, r \uparrow}$

$$
B^{3, r}=\frac{1}{\sqrt{3}}\left(b^{1, r}+b^{2, r}+b^{3, r}\right), \quad r=L, R
$$

and the other one depending on $\left(B^{r \dagger}, \bar{B}^{r \dagger}\right)$

$$
\begin{aligned}
& B^{r}=\frac{1}{\sqrt{3}}\left(e b^{1, r}+\bar{e} b^{2, r}+b^{3, r}\right), \quad r=L, R \\
& \bar{B}^{r}=\frac{1}{\sqrt{3}}\left(\bar{e} b^{1, r}+e b^{2, r}+b^{3, r}\right), \quad r=L, R
\end{aligned}
$$

Notice that in this notation we have $B_{n}^{r \dagger}=\bar{B}_{-n}^{r}$ and $\bar{B}_{n}^{r \dagger}=B_{-n}^{r}$ (where the usual convention $b_{-n}=b_{n}^{\dagger}$ applies). Observe that the first of these equations is identical to the overlap equation for the identity vertex. Hence, the comma 3-Vertex takes the form

$$
\begin{aligned}
\left|V_{Q}^{H S}\right\rangle= & \int \mathrm{d} Q_{M} \mathrm{~d} \bar{Q}_{M} \mathrm{~d} Q_{M}^{3} \delta\left(Q_{M}\right) \delta\left(\bar{Q}_{M}\right) \mathrm{e}^{i P_{M}^{3} Q_{M}^{3}} \\
& \times \mathrm{e}^{-\frac{1}{2}\left(B^{3 \dagger}|c| B^{3 \dagger}\right)-\left(B^{\dagger}|H| B^{\bar{\dagger}}\right)} \prod_{r=L, R}|0\rangle^{3, r}|0\rangle^{r}|\overline{0}\rangle^{r}
\end{aligned}
$$

where $C$ and $H$ are infinite dimensional matrices computed in [6] and the integration over $Q_{M}^{3}$ gives $\delta\left(P_{M}^{3}\right)$. However $P_{M}^{3}=P_{0}^{3}$ and so $\delta\left(P_{M}^{3}\right)$ is the statements of conservation of momentum at the center of mass of the three strings. Notice that the comma three interaction vertex separates into a product of two pieces as anticipated. The vacuum of the three strings, i.e., $\prod_{j=1}^{3}|0\rangle^{j, L}|0\rangle^{j, R}$, is however invariant under the $Z_{3}$-Fourier transformation. Thus we have $\prod_{r=1}^{2}|0\rangle^{3, r}|0\rangle^{r}|\overline{0}\rangle^{r}=\prod_{j=1}^{3}|0\rangle^{j, L}|0\rangle^{j, R}$. If we choose to substitute the explicit values of the matrices, the above expression reduces to the simple form

$$
\left|V_{x}^{H S}\right\rangle=\int \prod_{i=1}^{3} \mathrm{~d} x_{M}^{i} \delta\left(x_{M}^{i}-x_{M}^{i-1}\right) \delta\left(\sum_{j=1}^{3} p_{M}^{j}\right) \times \mathrm{e}^{-\sum_{j=1}^{3} \sum_{n=1}^{\infty} b_{n}^{j, L b_{n}^{+}} b_{n}^{j-1, R \dagger}}|0\rangle_{123}^{L}|0\rangle_{123}^{R}
$$


where $|0\rangle_{123}^{L, R}$ denotes the vacuum in the left (right) product of the Hilbert space of the three strings. Here $b_{n}^{j, L(R)}$ denotes oscillators in the $L(R) j$ th string Hilbert space. For simplicity the Lorentz index $(\mu=0,1, \cdots, 25)$ and the Minkowski metric $\eta_{\mu v}$ used to contract the Lorentz indices, have been suppressed in Equation (40). We shall follow this convention throughout this paper.

Though the form of the comma 3-Vertex given in Equation (40) is quite elegant, it is very cumbersome to relate it directly to the SCSV 3-Vertex due to the fact that connection between the vacuum in the comma theory and the vacuum in the $S C S V$ is quite involved. One also needs to use the change of representation formulas [1] to recast the quadratic form in the half string creation operators in terms of the full string creation-annihilation operators which adds more complications to an already difficult problem. On the other hand the task could be greatly simplified if we express the comma vertex in the full string basis. This may be achieved simply by re expressing the comma overlaps in terms of overlaps in the full string basis. Moreover, the proof of the Ward-like identities will also simplify a great deal if the comma 3-Vertex is expressed in the full string basis. Before we express the half-string 3-Vertex is expressed in the full string basis, we need first to solve the comma overlap equations in (27), (30) and (32), (34) for the Fourier modes of the comma coordinates and momenta, respectively. The modes in the $Z_{3}$ Fourier space are given by

$$
\begin{aligned}
& Q_{2 n-1}^{r}=\frac{1}{\pi \sqrt{2}} \int_{-\pi}^{\pi} Q^{r}(\sigma) \cos (2 n-1) \sigma \mathrm{d} \sigma, \\
& \bar{Q}_{2 n-1}^{r}=\frac{1}{\pi \sqrt{2}} \int_{-\pi}^{\pi} \bar{Q}^{r}(\sigma) \cos (2 n-1) \sigma \mathrm{d} \sigma, \\
& Q_{2 n-1}^{3, r}=\frac{1}{\pi \sqrt{2}} \int_{-\pi}^{\pi} Q^{3, r}(\sigma) \cos (2 n-1) \sigma \mathrm{d} \sigma
\end{aligned}
$$

where $n=1,2,3, \cdots$, and a similar set for the conjugate momenta. The overlap equations for the coordinates in (27) and (28) and the properties imposed in the Fourier expansion of the comma coordinates

$$
\begin{gathered}
Q^{r}(\sigma)=Q^{r}(-\sigma) \text { and } Q^{r}(\sigma)=-Q^{r}(\pi-\sigma), \\
\bar{Q}^{r}(\sigma)=\bar{Q}^{r}(-\sigma) \text { and } \bar{Q}^{r}(\sigma)=-\bar{Q}^{r}(\pi-\sigma), \\
Q^{3, r}(\sigma)=Q^{3, r}(-\sigma) \text { and } Q^{3, r}(\sigma)=-Q^{3, r}(\pi-\sigma)
\end{gathered}
$$

where $0 \leq \sigma \leq \pi$, imply that their $Z_{3}$ Fourier modes in the comma basis satisfy

$$
\begin{gathered}
Q_{2 n-1}^{L}=e Q_{2 n-1}^{R}, \\
\bar{Q}_{2 n-1}^{L}=\bar{e} \bar{Q}_{2 n-1}^{R}
\end{gathered}
$$

From the overlap in (30) we obtain

$$
Q_{2 n-1}^{3, L}=Q_{2 n-1}^{3, R}
$$

For the Fourier modes of the conjugate momenta one obtains 


$$
\begin{gathered}
\wp_{2 n-1}^{L}=-e \wp_{2 n-1}^{R}, \\
\bar{\wp}_{2 n-1}^{L}=-\bar{e} \bar{\wp}_{2 n-1}^{R}
\end{gathered}
$$

and

$$
\wp_{2 n-1}^{3, L}=-\wp_{2 n-1}^{3, R},
$$

where $n=1,2,3, \cdots$. We see that the comma overlaps in the full string basis separates into a product of two pieces depending on

$$
A_{n}^{3 \dagger}=\frac{1}{\sqrt{3}}\left(a_{n}^{1 \dagger}+a_{n}^{2 \dagger}+a_{n}^{3 \dagger}\right)
$$

and on

$$
\begin{aligned}
& A_{n}^{\dagger} \equiv A_{n}^{1 \dagger}=\frac{1}{\sqrt{3}}\left(\bar{e} a_{n}^{1 \dagger}+e a_{n}^{2 \dagger}+a_{n}^{3 \dagger}\right), \\
& \bar{A}_{n}^{\dagger} \equiv A_{n}^{2 \dagger}=\frac{1}{\sqrt{3}}\left(e a_{n}^{1 \dagger}+\bar{e} a_{n}^{2 \dagger}+a_{n}^{3 \dagger}\right),
\end{aligned}
$$

respectively, where the creation and annihilation operators $A_{n}^{\dagger}$ and $A_{n}$ in the $Z_{3}$-Fourier space are defined in the usual way

$$
\begin{gathered}
Q_{n}=\frac{i}{2} \sqrt{\frac{2}{n}}\left(A_{n}-A_{n}^{\dagger}\right), n=1,2,3, \cdots \\
Q_{0}=\frac{i}{2}\left(A_{0}-A_{0}^{\dagger}\right) \\
P_{n}=-i \frac{\partial}{\partial Q_{n}}=\sqrt{\frac{n}{2}}\left(A_{n}+A_{n}^{\dagger}\right), n=1,2,3, \cdots \\
P_{0}=-i \frac{\partial}{\partial Q_{0}}=\left(A_{0}+A_{0}^{\dagger}\right)
\end{gathered}
$$

and similarly for $\bar{A}_{n}^{\dagger}, \bar{A}_{n}$ and $A_{n}^{3 \dagger}, A_{n}^{3}$. Notice that in the $Z_{3}$-Fourier space, $A_{n}^{\dagger}=\bar{A}_{-n}, \quad \bar{A}_{n}^{\dagger}=A_{-n}$. For the matter sector, the comma 3 -Vertex would be represented as exponential of quadratic form in the creation operators $A_{n}^{3 \dagger}, A_{n}^{\dagger}$ and $\bar{A}_{m}^{\dagger}$. Thus the comma 3-Vertex in the full string $Z_{3}$-Fourier space takes the form

$$
\left.\left|V_{Q}^{H S}\right\rangle=\int \mathrm{d} Q_{M} \mathrm{~d} \bar{Q}_{M} \delta\left(Q_{M}\right) \delta\left(\bar{Q}_{M}\right) V^{H S}\left(A_{n}^{3 \dagger}, A_{n}^{\dagger}, \bar{A}_{n}^{\dagger}\right) \mid 0\right)_{123}
$$

where $\mid 0)_{123}$ denotes the matter part of the vacuum in the Hilbert space of the three strings and

$$
V^{H S}\left(A_{n}^{3 \dagger}, A_{n}^{\dagger}, \bar{A}_{n}^{\dagger}\right)=\mathrm{e}^{\sum_{n, m=0}^{\infty}\left(-\frac{1}{2} A_{n}^{3 \dagger} C_{n m} A_{m}^{3 \dagger}-A_{n}^{\dagger} F_{n m} \bar{A}_{m}^{\dagger}\right)}
$$

The ghost piece of the 3-Vertex in the bosonized form has the same structure as the coordinate piece apart from the mid point insertions. In the $Z_{3}$-Fourier space $Q_{M}^{\phi}={\overline{Q^{\phi}}}_{M}=0$ and only $Q_{M}^{\phi 3} \neq 0$. Thus the mid-point insertion is given by $3 i Q_{M}^{\phi 3} / 2$. The effect of the insertion is to inject the ghost number into the vertex at its mid-point to conserve the ghost number at the string mid-point, where the conservation of ghost number is violated due to the concentration of 
the curvature at the mid-point. Thus the ghost part of the 3 -Vertex takes the form

$$
\left.\left|V_{Q^{\phi}}^{H S}\right\rangle=\mathrm{e}^{3 i Q_{M}^{\phi, 3} / 2} V_{\phi}^{H S}\left(A_{n}^{\phi, 3 \dagger}, A_{n}^{\phi \dagger}, \overline{A^{\phi} \dagger}{ }_{n}\right) \mid 0\right)_{123}^{\phi}
$$

where $|0\rangle_{123}^{\phi}$ denotes the ghost part of the vacuum in the Hilbert space of the three strings and $V_{\phi}^{H S}\left(A_{n}^{\phi, 3 \dagger}, A_{n}^{\phi \dagger}, \bar{A}_{n}^{\phi \dagger}\right)$ has the exact structure as the coordinate piece $V_{Q}^{H S}\left(A_{n}^{3 \dagger}, A_{n}^{\dagger}, \bar{A}_{n}^{\dagger}\right)$. The mid-point insertion $3 i Q_{M}^{\phi, 3} / 2$ in (62) may be written in terms of the creation annihilation operators

$$
Q_{M}^{\phi, 3}=Q_{0}^{\phi, 3}+i \sum_{n=\text { even }=2}^{\infty} \frac{(-1)^{n / 2}}{\sqrt{n}}\left(A_{n}^{3}-A_{n}^{3 \dagger}\right)
$$

If we now commute the annihilation operators in the mid-point insertion through the exponential of the quadratic form in the creation operators in the three-string ghost vertex $\left(V_{Q^{\phi}}^{H S}\right)$, the three-string ghost vertex in (62) takes the form

$$
\left|V_{Q^{\phi}}^{H S}\right\rangle=\mathrm{e}^{3 i Q_{0}^{\phi, 3} / 2} \mathrm{e}^{3 \sum_{n=v e r n=2}^{\infty} \frac{(-1)^{n / 2}}{\sqrt{n}} A_{n}^{3 \dagger}} V_{\phi}^{H S}\left(A_{n}^{\phi, 3 \dagger}, A_{n}^{\phi \dagger}, \overline{A^{\phi \dagger}}{ }_{n}^{\dagger}\right)|0\rangle_{123}^{\phi}
$$

We note that commuting the annihilation operators in the mid-point insertion $3 i Q_{M}^{\phi, 3} / 2$ through $V_{\phi}^{H S}\left(A_{n}^{\phi, 3 \dagger}, A_{n}^{\phi \dagger}, \overline{A^{\phi} \dagger}\right)$ results in the doubling of the creation operator in the mid-point insertion.

\section{The Half-String 3-Vertex in the Full String Basis}

We now proceed to express the half-string overlaps in the Hilbert space of the full string theory. The change of representation between the half-string modes and the full string modes derived in [1] is given by

$$
\begin{aligned}
& Q_{n}^{r}=(-1)^{r+1} Q_{2 n-1}+\sum_{m=1}^{\infty} \sqrt{\frac{2 m}{2 n-1}}\left[M_{m n}^{1}+M_{m n}^{2}\right] Q_{2 m} \\
& \wp_{n}^{r}=\frac{(-1)^{r+1}}{2} P_{2 n-1}+\frac{1}{2} \sum_{m=1}^{\infty} \sqrt{\frac{2 n-1}{2 m}}\left[M_{m n}^{1}-M_{m n}^{2}\right] P_{2 m}-\frac{\sqrt{2}}{\pi} \frac{(-1)^{n}}{2 n-1} P_{0}
\end{aligned}
$$

where $r=1,2 \equiv L, R ; n=1,2,3, \cdots$; and the matrices $M^{1}$ and $M^{2}$ are given by

$$
M_{m n}^{1}=\frac{2}{\pi} \sqrt{\frac{2 m}{2 n-1}} \frac{(-1)^{m+n}}{2 m-(2 n-1)}, m, n=1,2,3, \cdots
$$

and

$$
M_{m n}^{2}=\frac{2}{\pi} \sqrt{\frac{2 m}{2 n-1}} \frac{(-1)^{m+n}}{2 m+(2 n-1)}, m, n=1,2,3, \cdots
$$

Now the overlap equations in (47), (50) and (29) become

$$
(1+e) Q_{2 n-1}=-(1-e) \sum_{m=1}^{\infty} \sqrt{\frac{2 m}{2 n-1}}\left[M_{m n}^{1}+M_{m n}^{2}\right] Q_{2 m}
$$




$$
\begin{gathered}
(1-e) \frac{1}{2} P_{2 n-1}=-(1+e) \frac{1}{2} \sum_{m=1}^{\infty} \sqrt{\frac{2 n-1}{2 m}}\left[M_{m n}^{1}-M_{m n}^{2}\right] P_{2 m}+(1+e) \frac{\sqrt{2}}{\pi} \frac{(-1)^{n}}{2 n-1} P_{0} \\
Q_{M}=Q_{0}+\sqrt{2} \sum_{n=1}^{\infty}(-1)^{n} Q_{2 n}=0
\end{gathered}
$$

respectively. The overlaps for the complex conjugate of the first two equations could be obtained simply by taking the complex conjugation. Similarly from the overlaps in (49), (52) and (35) we obtain

$$
\begin{gathered}
Q_{2 n-1}^{3}=0 \\
\sum_{m=1}^{\infty} \sqrt{\frac{2 n-1}{2 m}}\left[M_{m n}^{1}-M_{m n}^{2}\right] P_{2 m}^{3}-\frac{2 \sqrt{2}}{\pi} \frac{(-1)^{n}}{2 n-1} P_{0}^{3}=0 \\
\wp_{M}^{3}=0
\end{gathered}
$$

We have seen in reference [1] the $\wp_{M}^{3}=P_{0}^{3}$ and so the overlap conditions in (72) and (73) reduce to

$$
\begin{gathered}
\sum_{m=1}^{\infty} \sqrt{\frac{2 n-1}{2 m}}\left[M_{m n}^{1}-M_{m n}^{2}\right] P_{2 m}^{3}=0 \\
P_{0}^{3}=0
\end{gathered}
$$

It is important to keep in mind that the equality sign appearing in Equations (68) through (75) is an equality between action of the operators when acting on the comma vertex except for Equation (75) which is the conservation of the momentum carried by the third string in the $Z_{3}$ Fourier space.

The comma vertex $\left|V^{H S}\left(A_{n}^{3 \dagger}, A_{n}^{\dagger}, \bar{A}_{n}^{\dagger}\right)\right\rangle$ in the full string basis now satisfies the comma overlaps in (68), (69), (70), (71), (74) and (74). First let us consider the overlaps in (68), (69) and (70), i.e.,

$$
\begin{aligned}
& {\left[(1+e) Q_{2 n-1}+(1-e) \sum_{m=1}^{\infty} \sqrt{\frac{2 m}{2 n-1}}\left(M_{m n}^{1}+M_{m n}^{2}\right) Q_{2 m}\right]} \\
& \left|V^{H S}\left(A_{n}^{3 \dagger}, A_{n}^{\dagger}, \bar{A}_{n}^{\dagger}\right)\right\rangle=0, \\
& {\left[(1-e) \frac{1}{2} P_{2 n-1}+(1+e) \frac{1}{2} \sum_{m=1}^{\infty} \sqrt{\frac{2 n-1}{2 m}}\left(M_{m n}^{1}-M_{m n}^{2}\right) P_{2 m}\right.} \\
& \left.-(1+e) \frac{\sqrt{2}}{\pi} \frac{(-1)^{n}}{2 n-1} P_{0}\right]\left|V^{H S}\left(A_{n}^{3 \dagger}, A_{n}^{\dagger}, \bar{A}_{n}^{\dagger}\right)\right\rangle=0, \\
& {\left[Q_{0}+\sqrt{2} \sum_{k=1}^{\infty}(-1)^{k} Q_{2 k}\right]\left|V^{H S}\left(A_{n}^{3 \dagger}, A_{n}^{\dagger}, \bar{A}_{n}^{\dagger}\right)\right\rangle=0}
\end{aligned}
$$

(as well as their complex conjugates), where $n=1,2,3, \cdots$. For the remaining overlaps, i.e., equations in (71) and (74), we have

$$
\begin{gathered}
Q_{2 n-1}^{3}\left|V^{H S}\left(A_{n}^{3 \dagger}, A_{n}^{\dagger}, \bar{A}_{n}^{\dagger}\right)\right\rangle=0, \\
\sum_{m=1}^{\infty} \sqrt{\frac{2 n-1}{2 m}}\left(M_{m n}^{1}-M_{m n}^{2}\right) P_{2 m}^{3}\left|V^{H S}\left(A_{n}^{3 \dagger}, A_{n}^{\dagger}, \bar{A}_{n}^{\dagger}\right)\right\rangle=0,
\end{gathered}
$$




$$
P_{0}^{3}\left|V^{H S}\left(A_{n}^{3 \dagger}, A_{n}^{\dagger}, \bar{A}_{n}^{\dagger}\right)\right\rangle=0
$$

where $n=1,2,3, \cdots$. We notice that these overlaps are identical to the overlap equations for the identity vertex [4] [5] [7] [8]. Thus

$$
C_{n m}=(-1)^{n} \delta_{n m}, \quad n, m=0,1,2, \cdots
$$

The explicit form of the matrix $F$, may be obtained from the overlap equations given by (76), (77) and (78) as well as their complex conjugates. It will turn out that the matrix $F$ has the following properties

$$
F=F^{\dagger}, \quad \bar{F}=C F C, \quad F^{2}=1
$$

which are consistent with the properties of the coupling matrices in Witten's theory of open bosonic strings [7] [8]. This indeed is a nontrivial check on the validity of the comma approach to the theory of open bosonic strings.

Now substituting (61) into (76) and writing $Q_{n}$ in terms of $A_{n}^{\dagger}$ and $A_{n}$, we obtain the first equation for the matrix $F$

$$
F_{2 n-1 k}+\delta_{2 n-1 k}-i \sqrt{3} \sum_{m=1}^{\infty}\left(M_{m n}^{1}+M_{m n}^{2}\right)\left(F_{2 m k}+\delta_{2 m k}\right)=0
$$

where $k=0,1,2, \cdots ; n=1,2,3, \cdots$. Next from the overlap equation in (77) we obtain a second condition on the $F$ matrix

$$
\begin{aligned}
0= & \left(F_{2 n-1 k}-\delta_{2 n-1 k}\right)+\frac{1}{\sqrt{3}} i \sum_{m=1}^{\infty}\left(M_{m n}^{1}-M_{m n}^{2}\right)\left(F_{2 m k}-\delta_{2 m k}\right) \\
& -\frac{4}{\pi} \frac{i}{\sqrt{3}} \frac{(-1)^{n}}{(2 n-1)^{3 / 2}}\left(F_{0 k}-\delta_{0 k}\right)
\end{aligned}
$$

where $k=0,1,2, \cdots ; n=1,2,3, \cdots$. The overlaps for the mid-point in (78) give

$$
\left[\left(F_{0 m}+\delta_{0 m}\right)+\sqrt{2} \sum_{k=1}^{\infty}(-1)^{k} \sqrt{\frac{2}{2 k}}\left(F_{2 k m}+\delta_{2 k m}\right)\right]=0, m=0,1,2, \cdots
$$

Solving Equations (84) and (85), we have

$$
\begin{aligned}
& F_{2 n 0}= \frac{1}{\pi}\left(F_{00}-1\right) \sum_{m=1}^{\infty}\left[\left(M_{1}^{T}+\frac{1}{2} M_{2}^{T}\right)^{-1}\right]_{n m} \frac{(-1)^{m}}{(2 m-1)^{3 / 2}} \\
& F_{2 n 2 k}= \frac{1}{\pi} F_{02 k} \sum_{m=1}^{\infty}\left[\left(M_{1}^{T}+\frac{1}{2} M_{2}^{T}\right)^{-1}\right]_{n m} \frac{(-)^{m}}{(2 m-1)^{3 / 2}} \\
&-\sum_{m=1}^{\infty}\left[\left(M_{1}^{T}+\frac{1}{2} M_{2}^{T}\right)^{-1}\right]\left[\frac{1}{2} M_{1}^{T}+M_{2}^{T}\right]_{m k} \\
& F_{2 n 2 k-1}=-\frac{i \sqrt{3}}{2}\left[\left(M_{1}^{T}+\frac{1}{2} M_{2}^{T}\right)^{-1}\right]_{n k} \\
&+\frac{1}{\pi} F_{02 k-1} \sum_{m=1}^{\infty}\left[\left(M_{1}^{T}+\frac{1}{2} M_{2}^{T}\right)^{-1}\right] \frac{(-)^{m}}{(2 m-1)^{3 / 2}} \\
& F_{2 n-12 k-1}=\frac{2 i}{\sqrt{3}} \sum_{m=1}^{\infty}\left[\frac{1}{2} M_{1}^{T}+M_{2}^{T}\right]_{n m} F_{2 m 2 k-1}+\frac{2 i}{\pi \sqrt{3}} \frac{(-)^{n}}{(2 n-1)^{3 / 2}} F_{02 k-1}
\end{aligned}
$$




$$
\begin{aligned}
F_{2 n-12 k}= & \frac{2 i}{\sqrt{3}} \sum_{m=1}^{\infty}\left[\frac{1}{2} M_{1}^{T}+M_{2}^{T}\right]_{n m} F_{2 m 2 k}+\frac{2 i}{\sqrt{3}}\left[M_{1}^{T}+\frac{1}{2} M_{2}^{T}\right]_{n k} \\
& +\frac{2 i}{\pi \sqrt{3}} \frac{(-1)^{n}}{(2 n-1)^{3 / 2}} F_{02 k} \\
F_{2 n-10}= & \frac{2 i}{\sqrt{3}} \sum_{m=1}^{\infty}\left[\frac{1}{2} M_{1}^{T}+M_{2}^{T}\right]_{n m} F_{2 m 0}+\frac{2 i}{\pi \sqrt{3}} \times \frac{(-)^{n}}{(2 n-1)^{3 / 2}}\left(F_{00}-1\right)
\end{aligned}
$$

where all $n, k=1,2,3, \cdots$. Finally Equation (86) leads to

$$
\begin{gathered}
\left(F_{00}+1\right)=2 \sum_{n=1}^{\infty} \frac{(-1)^{n+1}}{\sqrt{2 n}} F_{2 n 0}, \\
F_{02 m}=2 \frac{(-1)^{m+1}}{\sqrt{2 m}}+2 \sum_{k=1}^{\infty} \frac{(-1)^{k+1}}{\sqrt{2 k}} F_{2 k 2 m}, \\
F_{02 m-1}=2 \sum_{k=1}^{\infty} \frac{(-1)^{k+1}}{\sqrt{2 k}} F_{2 k 2 m-1}
\end{gathered}
$$

where $m=1,2,3, \cdots$.

Now the explicit form of the $F$ matrix is completely given by the set of Equations (87), (88), (89), (90), (91), (92), (93), (94) and (95) provided that the inverse of the $\left(M_{1}^{T}+\frac{1}{2} M_{2}^{T}\right)$ exist. Now we proceed to compute the required inverse.

\section{Finding the Inverse}

In the half string formulation the combination $\left(M_{1}^{T}+\frac{1}{2} M_{2}^{T}\right)^{-1}$ is a special case of the more general expression, $M_{1}^{T}+\cos (k \pi / N) M_{2}^{T}$, where $k=1,2,3, \cdots, 2 N$ and $N$ is the number of strings ${ }^{2}$. For the case of interest, $N$ corresponds to 3 and $k=1$. It is however more constructive to consider the generic combination $\beta M_{1}^{T}+\alpha M_{2}^{T}$. Again for the case of interest one has $\beta=1$ and $\alpha=\cos (k \pi / N)=1 / 2$. For the inverse of $\beta M_{1}^{T}+\alpha M_{2}^{T}$, we propose the Ansatz

$$
\begin{aligned}
& {\left[\left(\beta M_{1}^{T}+\alpha M_{2}^{T}\right)^{-1}\right]_{n m}} \\
& =(-1)^{n+m} \sqrt{2 n}\left[\alpha^{\prime} \frac{u_{2 n}^{1-1 / p} u_{2 m-1}^{1 / p}+u_{2 n}^{1 / p} u_{2 m-1}^{1-1 / p}}{2 n-(2 m-1)}+\beta^{\prime} \frac{u_{2 n}^{1-1 / p} u_{2 m-1}^{1 / p}-u_{2 n}^{1 / p} u_{2 m-1}^{1-1 / p}}{2 n+(2 m-1)}\right] \sqrt{2 m-1}
\end{aligned}
$$

The coefficients $u_{k}^{1 / p}$ and $u_{k}^{1-1 / p}$ are the modes appearing in the Taylor expansion of the functions $\left(\frac{1+x}{1-x}\right)^{1 / p}$ and $\left(\frac{1+x}{1-x}\right)^{1-1 / p}$ respectively. For the three interaction vertex $p=3$ and the Taylor modes $u_{k}^{1 / p}$ and $u_{k}^{1-1 / p}$ reduce to $u_{k}^{1 / 3}=a_{k}$ and $u_{k}^{2 / 3}=b_{k}$ found in references [7] [8]. These coefficients are ${ }^{2}$ The reason we are considering this more general expression is that this combination appears in computing the $N$-interaction vertex which will probe useful in future work and it does not add to the level of difficulty in finding the inverse. 
treated in details in appendix A. The free parameters $\alpha^{\prime}, \beta^{\prime}$ and $p$ are to be determined by demanding that (96) satisfies the identities

$$
\left(\beta M_{1}^{T}+\alpha M_{2}^{T}\right)^{-1}\left(\beta M_{1}^{T}+\alpha M_{2}^{T}\right)=I
$$

which implies that $\left(\beta M_{1}^{T}+\alpha M_{2}^{T}\right)^{-1}$ is left inverse and the identity

$$
\left(\beta M_{1}^{T}+\alpha M_{2}^{T}\right)\left(\beta M_{1}^{T}+\alpha M_{2}^{T}\right)^{-1}=I
$$

which implies that $\left(\beta M_{1}^{T}+\alpha M_{2}^{T}\right)^{-1}$ is right inverse. Here $I$ is the identity matrix in the space of $N$ strings.

Before we proceed to fix the constants $\alpha^{\prime}, \beta^{\prime}$ and $p$, there are two special cases where the inverse could be obtained with ease with the help of the commutation relations of the half string creation annihilation operators $\left(b_{n}^{(r)}, b_{n}^{(r) \dagger}\right)$. They are given by $k=2 N$ and $k=N$.

For $k=2 N$, the combination $M_{1}^{T}+\cos (k \pi / N) M_{2}^{T}$ reduces to $M_{1}^{T}+M_{2}^{T}$ and the inverse $\left(M_{1}^{T}+M_{2}^{T}\right)^{-1}=M_{1}-M_{2}$. To see this we only need to verify that $\left(M_{1}^{T}+M_{2}^{T}\right)\left(M_{1}-M_{2}\right)=\left(M_{1}-M_{2}\right)\left(M_{1}^{T}+M_{2}^{T}\right)=I$. We first consider

$$
\left(M_{1}^{T}+M_{2}^{T}\right)\left(M_{1}-M_{2}\right)=\left(M_{1}^{T} M_{1}-M_{2}^{T} M_{2}\right)-\left(M_{1}^{T} M_{2}-M_{2}^{T} M_{1}\right)
$$

Using the commutation relations

$$
\left[b_{n}^{(r)}, b_{-m}^{(s)}\right]=\delta^{r s} \delta_{n+m 0}
$$

(where $b_{-m}^{(s)} \equiv b_{m}^{(s) \dagger}$ ) for the half string creation annihilation modes $\left(b_{n}^{(r)}, b_{n}^{(r) \dagger}\right)$, one can show that the the combination inside the first bracket is the identity matrix $I$ and the combination inside the second bracket is identically zero. To see this recall that the change of representation between the full string creation annihilation modes $\left(a_{n}, a_{n}^{\dagger}\right)$ and the half string creation annihilation modes is given by

$$
b_{n}^{(r)}=(-1)^{r} a_{2 n-1}+\frac{1}{2} \sum_{m=1}^{\infty}\left(M_{m n}^{1} a_{2 m}-M_{m n}^{2} a_{-2 m}\right), n=1,2,3, \cdots
$$

and $b_{-n}^{(r)} \equiv b_{n}^{(r) \dagger}$ is given by the same expression with $a_{k} \rightleftharpoons a_{-k}$. Substituting (101) into (100) one obtains

$$
\begin{aligned}
0 & =\left[b_{n}^{(r)}, b_{q}^{(s)}\right]=-\frac{1}{2} \delta^{r s} \sum_{k=1}^{\infty}\left[\left(M_{1}^{T}\right)_{n k}\left(M_{2}\right)_{k q}-\left(M_{2}^{T}\right)_{n k}\left(M_{1}\right)_{k q}\right] \\
& =-\frac{1}{2} \delta^{r s}\left[M_{1}^{T} M_{2}-M_{2}^{T} M_{1}\right]_{n q}
\end{aligned}
$$

for $n>0$ and $m=-q<0$. Since $\delta^{r s}=0$ for $r \neq s$, then the above equation does not yield any information about the combination $M_{1}^{T} M_{2}-M_{2}^{T} M_{1}$ for $r \neq s$. However, for $r=s$, Equation (102) yields

$$
M_{1}^{T} M_{2}-M_{2}^{T} M_{1}=0
$$

Similarly one has

$$
\begin{aligned}
\delta^{r s} \delta_{n m} & =\left[b_{n}^{(r)}, b_{-m}^{(s)}\right] \\
& =\frac{1}{2}(-1)^{r+s} \delta_{n m}+\frac{1}{2} \sum_{m=1}^{\infty}\left[\left(M_{1}^{T}\right)_{n k}\left(M_{1}\right)_{k m}-\left(M_{2}^{T}\right)_{n k}\left(M_{2}\right)_{k m}\right] \\
& =\frac{1}{2}(-1)^{r+s} I_{n m}+\frac{1}{2}\left[M_{1}^{T} M_{1}-M_{2}^{T} M_{2}\right]_{n m}
\end{aligned}
$$


for $n, m>0$. In this case, the above expression gives the following identity

$$
M_{1}^{T} M_{1}-M_{2}^{T} M_{2}=I
$$

for all possible values of $r$ and $s$. Substituting Equations (103) and (105) into Equation (99) we arrive at

$$
\left(M_{1}^{T}+M_{2}^{T}\right)\left(M_{1}-M_{2}\right)=I
$$

Thus $\left(M_{1}-M_{2}\right)$ is the right inverse of $\left(M_{1}^{T}+M_{2}^{T}\right)$. To complete the proof one must show that $\left(M_{1}-M_{2}\right)$ is also a left inverse; that is we need to establish the following identity

$$
\left(M_{1}-M_{2}\right)\left(M_{1}^{T}+M_{2}^{T}\right)=I
$$

The proof of the above identity follows at once from the change of representation between the half string creation annihilation modes $\left(b_{n}^{(r)}, b_{n}^{(r) \dagger}\right)$ and the full string creation annihilation modes $\left(a_{n}, a_{n}^{\dagger}\right)$ given by

$$
a_{2 n}=\frac{(-1)^{n}}{\sqrt{2 n}} P+\sum_{m=1}^{\infty}\left(M_{n m}^{1} b_{m}^{(+)}-M_{n m}^{2} b_{-2 m}^{(+)}\right), \quad n=1,2,3, \cdots
$$

(where $\left.b_{n}^{(+)}=\frac{1}{\sqrt{2}}\left(b_{n}^{(1)}+b_{n}^{(2)}\right)\right)$ and the commutation relations

$$
\left[a_{n}, a_{-m}\right]=\delta_{n+m 0} .
$$

Using Equations (108) and (109) and skipping the algebraic details, one obtains the following identities

$$
\begin{aligned}
& M_{1} M_{2}^{T}-M_{2} M_{1}^{T}=0 \\
& M_{1} M_{1}^{T}-M_{2} M_{2}^{T}=I
\end{aligned}
$$

needed to prove that the combination $\left(M_{1}-M_{2}\right)$ is also a left inverse. This completes the proof.

For $k=N$, the combination $M_{1}^{T}+\cos (k \pi / N) M_{2}^{T}$ reduces to $M_{1}^{T}-M_{2}^{T}$ and the inverse $\left(M_{1}^{T}-M_{2}^{T}\right)^{-1}=M_{1}+M_{2}$. The proof that $M_{1}+M_{2}$ is the right inverse follows at once simply by taking the transpose of the already established identity in (106). To show that the combination $M_{1}+M_{2}$ is also the left inverse of the combination $M_{1}^{T}-M_{2}^{T}$ one only needs to take the transpose of (107); thus leading to the desired result.

Now we proceed to fix the constants in (96) for $k \neq N, 2 N$. From Equations (66) and (67), we have

$$
M_{n m}^{1}=\frac{2}{\pi} \sqrt{\frac{2 n}{2 m-1}} \frac{(-1)^{n+m}}{2 n-(2 m-1)}
$$

and

$$
M_{n m}^{2}=\frac{2}{\pi} \sqrt{\frac{2 n}{2 m-1}} \frac{(-1)^{n+m}}{2 n+(2 m-1)}
$$

respectively. First we proceed with the identity in (97). If we could solve for the free parameters $\alpha^{\prime}, \beta^{\prime}$, and $p$ in terms of the known parameters $\alpha$ and $\beta$ then the Ansatz in (96) is the left inverse of the matrix $\beta M_{1}^{T}+\alpha M_{2}^{T}$. For the off 
diagonal elements; that is $q \neq n$, the identity in (97), yields, after much use of the identities in [9],

$$
\begin{aligned}
& \alpha^{\prime} \beta \frac{u_{2 n}^{1-1 / p} O_{-2 n}^{u(1, p)}-u_{2 n}^{1-1 / p} O_{-2 q}^{u(1, p)}+u_{2 n}^{1 / p} O_{-2 n}^{u(p-1, p)}-u_{2 n}^{1 / p} O_{-2 q}^{u(p-1, p)}}{2 n-2 q} \\
& -\alpha^{\prime} \alpha \frac{u_{2 n}^{1-1 / p} O_{-2 n}^{u(1, p)}-u_{2 n}^{1-1 / p} O_{2 q}^{u(1, p)}+u_{2 n}^{1 / p} O_{-2 n}^{u(p-1, p)}-u_{2 n}^{1 / p} O_{2 q}^{u(p-1, p)}}{2 n+2 q} \\
& +\beta^{\prime} \beta \frac{u_{2 n}^{1-1 / p} O_{2 n}^{u(1, p)}-u_{2 n}^{1-1 / p} O_{-2 q}^{u(1, p)}-u_{2 n}^{1 / p} O_{2 n}^{u(p-1, p)}+u_{2 n}^{1 / p} O_{-2 q}^{u(p-1, p)}}{2 n+2 q} \\
& -\beta^{\prime} \alpha \frac{u_{2 n}^{1-1 / p} O_{2 n}^{u(1, p)}-u_{2 n}^{1-1 / p} O_{2 q}^{u(1, p)}-u_{2 n}^{1 / p} O_{2 n}^{u(p-1, p)}+u_{2 n}^{1 / p} O_{2 q}^{u(p-1, p)}}{2 n-2 q}=0
\end{aligned}
$$

where the quantities

$$
O_{ \pm n=2 k}^{u(q, p)} \equiv \sum_{m=2 l+1=1}^{\infty} \frac{u_{m}^{q / p}}{ \pm n+m}, n \geq 0
$$

have been considered in [9]. The quantities $O_{-n}^{u(q, p)}$ are related to $O_{n}^{u(q, p)}$ through the identity $O_{-n}^{u(q, p)}=-\cos (q \pi / p) O_{n}^{u(q, p)} \quad$ [9]. The quantity $O_{n}^{u(q, p)}$ has the value $[\pi / 2 \sin (q \pi / p)] u_{2 n}^{q / p} \quad$ [9]. In order for the right hand side of the above expression to vanish, the coefficients of $u_{2 n}^{1-1 / p} u_{2 n}^{1 / p}, u_{2 n}^{1-1 / p} u_{2 q}^{1 / p}$ and $u_{2 n}^{1 / p} u_{2 q}^{1-1 / p}$ must vanish separately. The vanishing of the coefficient of the $u_{2 n}^{1-1 / p} u_{2 n}^{1 / p}$ can be established explicitly by substituting the explicit values for $O_{n}^{u(q, p)}$. The vanishing of the coefficient of $u_{2 n}^{1-1 / p} u_{2 q}^{1 / p}$ term leads to the following conditions on the free parameters

$$
\begin{aligned}
& \beta^{\prime} \alpha+\alpha^{\prime} \beta \cos \left(\frac{1}{p} \pi\right)=0 \\
& \alpha^{\prime} \alpha+\beta^{\prime} \beta \cos \left(\frac{1}{p} \pi\right)=0
\end{aligned}
$$

The vanishing of the coefficient of $u_{2 n}^{1 / p} u_{2 q}^{1-1 / p}$ does not lead to new conditions on the free parameters but it provides a consistency condition. The equivalence between the half-string field theory and Witten's theory of open bosonic strings will guarantee that this consistency condition will be met. In fact we have verified this requirement explicitly.

For the diagonal elements $(q=n)$, the identity in (97), after much use of the various identities in [9], yields

$$
\begin{aligned}
1= & \frac{2(-1)^{n+n}}{\pi}(2 n)^{1 / 2}(2 n)^{1 / 2}\left\{\alpha^{\prime} \beta\left[u_{2 n}^{1-1 / p} \tilde{O}_{-2 n}^{u(1, p)}+u_{2 n}^{1 / p} \tilde{O}_{-2 n}^{u(p-1, p)}\right]\right. \\
& -\alpha^{\prime} \alpha \frac{u_{2 n}^{1-1 / p} O_{-2 n}^{u(1, p)}-u_{2 n}^{1-1 / p} O_{2 n}^{u(1, p)}+u_{2 n}^{1 / p} O_{-2 n}^{u(p-1, p)}-u_{2 n}^{1 / p} O_{2 n}^{u(p-1, p)}}{2(2 n)} \\
& +\beta^{\prime} \beta \frac{u_{2 n}^{1-1 / p} O_{2 n}^{u(1, p)}-u_{2 n}^{1-1 / p} O_{-2 n}^{u(1, p)}-u_{2 n}^{1 / p} O_{2 n}^{u(p-1, p)}+u_{2 n}^{1 / p} O_{-2 n}^{u(p-1, p)}}{2(2 n)} \\
& \left.+\beta^{\prime} \alpha\left[u_{2 n}^{1-1 / p} \tilde{O}_{2 n}^{u(1, p)}-u_{2 n}^{1 / p} \tilde{O}_{2 n}^{u(p-1, p)}\right]\right\}
\end{aligned}
$$


where

$$
\tilde{O}_{ \pm n=2 k}^{u(q, p)}=\sum_{m=2 l+1=1}^{\infty} \frac{u_{m}^{q / p}}{( \pm n+m)^{2}}
$$

has been considered in [9]. Using the explicit values of $O_{n}^{u(q, p)}, O_{-n}^{u(q, p)}, \tilde{O}_{n}^{u(q, p)}$ and $\tilde{O}_{-n}^{u(q, p)}$ which are given in [9] and imposing the conditions obtained in (116), the above expression reduces, after a lengthy exercise, otherwise a straight forward algebra, to

$$
1=\frac{2}{\pi}(2 n) \beta^{\prime} \alpha\left[u_{2 n}^{1-1 / p} \tilde{S}_{2 n}^{(1, p)}-u_{2 n}^{1 / p} \tilde{S}_{2 n}^{(p-1, p)}\right]
$$

where the quantities $\tilde{S}_{ \pm n}^{(q, p)}$ were introduced in [9]. The above expression may be reduced further by expressing $\tilde{S}_{-n}^{(q, p)}$ in terms of $\tilde{S}_{n}^{(q, p)}$ through the relations

$$
\begin{gathered}
\tilde{S}_{-2 n}^{(1, p)}=\tilde{S}_{2 n}^{(1, p)} \cos \frac{\pi}{p}+\left[1+\cos \left(\frac{\pi}{p}\right)\right] \bar{S}_{0}^{(1, p)} S_{2 n}^{(1, p)} \\
\tilde{S}_{-2 n}^{(p-1, p)}=\tilde{S}_{2 n}^{(p-1, p)} \cos \frac{(p-1) \pi}{p}+\left[1+\cos \left(\frac{(p-1) \pi}{p}\right)\right] \bar{S}_{0}^{(p-1, p)} S_{2 n}^{(p-1, p)}
\end{gathered}
$$

which have been established in [9]. Hence

$$
\begin{aligned}
1= & \frac{2}{\pi}(2 n) \alpha^{\prime} \beta\left\{u_{2 n}^{1-1 / p}\left[1+\cos \left(\frac{\pi}{p}\right)\right] \bar{S}_{0}^{(1, p)} S_{2 n}^{(1, p)}\right. \\
& \left.+u_{2 n}^{1 / p}\left[1-\cos \left(\frac{\pi}{p}\right)\right] \bar{S}_{0}^{(p-1, p)} S_{2 n}^{(p-1, p)}\right\}
\end{aligned}
$$

In arriving at the above expression we used the fact that

$$
\alpha^{\prime} \beta \cos \left(\frac{(p-1)}{p} \pi\right)-\beta^{\prime} \alpha=\alpha^{\prime} \beta \cos \left(\frac{1}{p} \pi\right)+\beta^{\prime} \alpha=0
$$

Further simplification of (122) may be achieved by substituting the explicit values of $\bar{S}_{0}^{(1, p)}$ and $\bar{S}_{0}^{(p-1, p)}$ found in [9]. Thus Equation (122) reduces to

$$
\begin{aligned}
1= & 2 n \alpha^{\prime} \beta\left\{u_{2 n}^{1-1 / p}\left[1+\cos \left(\frac{\pi}{p}\right)\right] \tan \left(\frac{1}{p} \frac{\pi}{2}\right) S_{2 n}^{(1, p)}\right. \\
& \left.+S_{2 n}^{(1, p)}\left[1-\cos \left(\frac{\pi}{p}\right)\right] \cot \left(\frac{1}{p} \frac{\pi}{2}\right) S_{2 n}^{(p-1, p)}\right\} \\
= & 2 n \alpha^{\prime} \beta \sin \left(\frac{\pi}{p}\right)\left[u_{2 n}^{1-1 / p} S_{2 n}^{(1, p)}+S_{2 n}^{(1, p)} S_{2 n}^{(p-1, p)}\right]
\end{aligned}
$$

To compute the right-hand side of the above expression we need to evaluate the expression inside the square bracket. We will show that this expression has the explicit value $2 / 2 n$. Consider the matrix element defined by

$$
W_{m n}=\frac{u_{m}^{1 / p} u_{n}^{1-1 / p}+u_{m}^{1-1 / p} u_{n}^{1 / p}}{m+n}
$$

The matrix element $W_{m n}$ satisfies the following recursion relationship, which may be verified by direct substitution 


$$
0=(n+1) W_{n+1 m}-(n-1) W_{n-1 m}+(m+1) W_{n m+1}-(m-1) W_{n m-1}
$$

for $m+n=o d d$ integer. Letting $n \rightarrow 2 n-1 \geq 1, m \rightarrow 2 m \geq 2$ in (126), we obtain

$$
0=2 n W_{2 n 2 m}-(2 n-2) W_{2 n-22 m}+(2 m+1) W_{2 n-12 m+1}-(2 m-1) W_{2 n-12 m-1}
$$

Summing both sides of (127) over $m$, we have

$$
2 n \sum_{m=0}^{\infty} W_{2 n 2 m}-(2 n-2) \sum_{m=0}^{\infty} W_{2 n-22 m}=2 n W_{2 n 0}-(2 n-2) W_{2 n-20}+W_{2 n-11}
$$

Substituting the explicit values for $2 n W_{2 n 0}, W_{2 n-20}$ and $W_{2 n-11}$ into (128) we obtain

$$
\begin{aligned}
& 2 n \sum_{m=0}^{\infty} W_{2 n 2 m}-(2 n-2) \sum_{m=0}^{\infty} W_{2 n-22 m} \\
& =u_{2 n}^{1 / p}+u_{2 n}^{1-1 / p}-u_{2 n-2}^{1 / p}-u_{2 n-2}^{1-1 / p}+\frac{2}{2 n} u_{2 n-1}^{1 / p}-\frac{1}{2 n} \frac{2}{p} u_{2 n-1}^{1 / p}+\frac{1}{2 n} \frac{2}{p} u_{2 n-1}^{1-1 / p}
\end{aligned}
$$

Recalling the recursion relations for the Taylor modes established in [9]

$$
\begin{aligned}
u_{k+1}^{1 / p} & =\frac{1}{k+1}\left[\frac{2}{p} u_{k}^{1 / p}+(k-1) u_{k-1}^{1 / p}\right] \\
u_{k+1}^{1-1 / p} & =\frac{1}{k+1}\left[\frac{2}{p}(p-1) u_{k}^{1-1 / p}+(k-1) u_{k-1}^{1-1 / p}\right]
\end{aligned}
$$

If we now set $k=2 n-1$ in the recursion relations in (130) and (131) and then rearrange terms, we have

$$
\begin{gathered}
\frac{1}{2 n} \frac{2}{p} u_{2 n-1}^{1 / p}=u_{2 n}^{1 / p}-\frac{1}{2 n}(2 n-2) u_{2 n-2}^{1 / p}, \\
\frac{1}{2 n} \frac{2}{p} u_{2 n-1}^{1-1 / p}=-u_{2 n}^{1-1 / p}+\frac{2}{2 n} u_{2 n-1}^{1-1 / p}+\frac{2 n-2}{2 n} u_{2 n-2}^{1-1 / p}
\end{gathered}
$$

Substituting (132) and (133) in the above equations into (129), we find

$$
2 n \sum_{m=0}^{\infty} W_{2 n 2 m}=(2 n-2) \sum_{m=0}^{\infty} W_{2 n-22 m}
$$

Repeated application of the above identity implies that

$$
2 n \sum_{m=0}^{\infty} W_{2 n 2 m}=2 \sum_{m=0}^{\infty} W_{22 m}
$$

Substituting the explicit form of $W_{n m}$ into the above identity we have

$$
\left[u_{2 n}^{1 / p} S_{2 n}^{(p-1, p)}+u_{2 n}^{1-1 / p} S_{2 n}^{(1, p)}\right]=\frac{2}{2 n}\left[u_{2}^{1 / p} S_{2}^{(p-1, p)}+u_{2}^{1-1 / p} S_{2}^{(1, p)}\right]
$$

where

$$
\begin{gathered}
S_{n}^{(1, p)} \equiv \sum_{n+m=\text { even }, m=0}^{\infty} \frac{u_{m}^{1 / p}}{n+m}, \\
S_{n}^{(p-1, p)} \equiv \sum_{n+m=\text { even }, m=0}^{\infty} \frac{u_{m}^{1-1 / p}}{n+m}
\end{gathered}
$$

To complete the proof, it remains to show that the expression inside the 
square bracket on the right hand side of Equation (136) is equal to unity. This we do by explicit computation. Consider

$$
u_{2}^{1 / p} S_{2}^{(p-1, p)}+u_{2}^{1-1 / p} S_{2}^{(1, p)}
$$

Using the summation formulas for $S_{n}^{(1, p)}$ and $S_{n}^{(p-1, p)}$, which are given in [9], the above expression reduces to

$$
u_{2}^{1 / p} S_{2}^{(p-1, p)}+u_{2}^{1-1 / p} S_{2}^{(1, p)}=1
$$

and so Equation (136) yields

$$
\left[u_{2 n}^{1 / p} S_{2 n}^{(p-1, p)}+u_{2 n}^{1-1 / p} S_{2 n}^{(1, p)}\right]=\frac{2}{2 n}
$$

Substituting this result for the expression in the square bracket in (124) leads to one more condition on the parameters $\alpha^{\prime}$ and $p$

$$
\alpha^{\prime}=\frac{1}{2 \sin \left(\frac{1}{p} \pi\right) \beta}
$$

Collecting all the conditions on the free parameters, and then solving for the parameters $\alpha^{\prime}$ and $\beta^{\prime}$, and $p$ in terms of the known parameters $\alpha$ and $\beta$, we find

$$
\frac{\alpha^{2}}{\beta^{2}}=\cos ^{2}\left(\frac{1}{p} \pi\right), \alpha^{\prime}=\frac{1}{2 \sin \left(\frac{1}{p} \pi\right) \beta}, \beta^{\prime}=-\frac{\cos \left(\frac{1}{p} \pi\right)}{2 \sin \left(\frac{1}{p} \pi\right) \alpha}
$$

The desired expression for the inverse of $\beta M_{1}^{T}+\alpha M_{2}^{T}$, is therefore given by substituting the values of $\alpha^{\prime}$ and $\beta^{\prime}$ given by the above expressions into the Ansatz for $\left(\beta M_{1}^{T}+\alpha M_{2}^{T}\right)^{-1}$ in Equation (96). Hence,

$$
\begin{aligned}
& {\left[\left(\beta M_{1}^{T}+\alpha M_{2}^{T}\right)^{-1}\right]_{n m}} \\
& =(-1)^{n+m} \frac{\sqrt{2 n} \sqrt{2 m-1}}{2 \sin \left(\frac{1}{p} \pi\right)}\left[\frac{1}{\beta} \frac{u_{2 n}^{1-1 / p} u_{2 m-1}^{1 / p}+u_{2 n}^{1 / p} u_{2 m-1}^{1-1 / p}}{2 n-(2 m-1)}\right. \\
& \left.-\frac{\cos \left(\frac{1}{p} \pi\right)}{\alpha} \frac{u_{2 n}^{1-1 / p} u_{2 m-1}^{1 / p}-u_{2 n}^{1 / p} u_{2 m-1}^{1-1 / p}}{2 n+(2 m-1)}\right]
\end{aligned}
$$

This shows that the above expression is the left inverse. To complete the proof we need to check that the identity in (98) is also satisfied and leads to the same conditions as in Equations (143). This in fact we did verify. The special cases of $k=N$ and $k=2 N$ have been treated earlier. This completes the construction of the inverse for the general case of the $\left(\beta M_{1}^{T}+\alpha M_{2}^{T}\right)$ matrix.

In the particular case of $\left(M_{1}^{T}+\cos \frac{k \pi}{N} M_{2}^{T}\right)^{-1}$, the parameters 
$\alpha=\cos (k \pi / N)$ and $\beta=1$ respectively, and the above relations in (143) become

$$
\cos ^{2}\left(\frac{\pi}{p}\right)=\alpha^{2}=\cos ^{2}\left(\frac{k \pi}{N}\right), \alpha^{\prime}=\frac{1}{2 \sin \frac{\pi}{p}}, \beta^{\prime}=-\frac{\cos \frac{\pi}{p}}{2 \sin \frac{\pi}{p} \cos \frac{k \pi}{N}}
$$

For the particular case of $\alpha=\cos (k \pi / N)$ and $\beta=1$, the relations in (145) yield

$$
\begin{gathered}
\alpha^{\prime}=-\beta^{\prime}=\frac{1}{2 \sin \left(\frac{1}{p} \pi\right)} \\
1 / p= \begin{cases}k / N, & 1 \leq k \leq N-1 \\
(2 N-k) / N, & N+1 \leq k \leq 2 N-1\end{cases}
\end{gathered}
$$

If we choose $1 \leq k \leq N-1$, then we have

$$
\begin{aligned}
& {\left[\left(M_{1}^{T}+\cos \left(\frac{k \pi}{N}\right) M_{2}^{T}\right)^{-1}\right]_{n m}} \\
& =(-1)^{n+m} \frac{\sqrt{2 n} \sqrt{2 m-1}}{2 \sin \left(\frac{1}{p} \pi\right)}\left[\frac{u_{2 n}^{1-1 / p} u_{2 m-1}^{1 / p}+u_{2 n}^{1 / p} u_{2 m-1}^{1-1 / p}}{2 n-(2 m-1)}-\frac{u_{2 n}^{1-1 / p} u_{2 m-1}^{1 / p}-u_{2 n}^{1 / p} u_{2 m-1}^{1-1 / p}}{2 n+(2 m-1)}\right]
\end{aligned}
$$

For the case of interest, that is, the three interaction vertex $N=3$ and $k=1$ so that $p=3$. This implies that the Taylor modes $u_{n}^{1 / p}$ and $u_{n}^{1-1 / p}$ in the expansion of $\left(\frac{1+x}{1-x}\right)^{1 / p}$ and $\left(\frac{1+x}{1-x}\right)^{1-1 / p}$ are $a_{n}$ and $b_{n}$ in the expansion of $\left(\frac{1+x}{1-x}\right)^{1 / 3}$ and $\left(\frac{1+x}{1-x}\right)^{2 / 3}$ encountered in reference [7] [8]. Thus the inverse of $\left(M_{1}^{T}+\frac{1}{2} M_{2}^{T}\right)$ now reads

$$
\begin{aligned}
& {\left[\left(M_{1}^{T}+\frac{1}{2} M_{2}^{T}\right)^{-1}\right]_{n m}} \\
& =\frac{1}{\sqrt{3}}(-1)^{n+m} \sqrt{2 n} \sqrt{2 m-1}\left[\frac{a_{2 n} b_{2 m-1}+b_{2 n} a_{2 m-1}}{2 n-(2 m-1)}+\frac{a_{2 n} b_{2 m-1}-b_{2 n} a_{2 m-1}}{2 n+(2 m-1)}\right]
\end{aligned}
$$

This is the required inverse needed to finish the construction of the half-string three interaction vertex in terms of the full-string basis. The expression in (149) is indeed the right and left inverse of $M_{1}^{T}+\frac{1}{2} M_{2}^{T}$ as can be checked explicitly. See ref. [9].

\section{Computing the Explicit Values of the Matrix Elements of the $F$ Matrix}

To complete the construction of the comma 3-Vertex

$$
\left|V^{H S}\right\rangle=\int \mathrm{d} Q_{M}^{3} \mathrm{~d} Q_{M} \mathrm{~d} \bar{Q}_{M} \delta\left(Q_{M}\right) \delta\left(\bar{Q}_{M}\right) \mathrm{e}^{i P_{0}^{3} Q_{M}^{3}} V^{H S}\left(A_{n}^{3 \dagger}, A_{n}^{\dagger}, \bar{A}_{n}^{\dagger}\right)|0,0,0\rangle
$$


in the $Z_{3}$-Fourier space of the full string, we need the explicit form of the $F$ matrix. Here we shall give the steps involved in the computation of the matrix elements of $F$ and relegate many of the technical details to appendix A. For the purpose of illustration consider $F_{2 n 0}$. Substituting the explicit value of $\left(M_{1}^{T}+\frac{1}{2} M_{2}^{T}\right)^{-1}$ obtained in (149) into Equation (87) gives

$$
\begin{aligned}
F_{2 n 0}= & \frac{1}{\pi}\left(F_{00}-1\right) \sum_{m=1}^{\infty} \frac{1}{\sqrt{3}}(-1)^{n+m} \sqrt{2 n} \sqrt{2 m-1} \cdot \frac{(-1)^{m}}{(2 m-1)^{3 / 2}} \\
& \times\left[\frac{a_{2 n} b_{2 m-1}+b_{2 n} a_{2 m-1}}{2 n-(2 m-1)}+\frac{a_{2 n} b_{2 m-1}-b_{2 n} a_{2 m-1}}{2 n+(2 m-1)}\right]
\end{aligned}
$$

where $n=1,2,3, \cdots$. Using partial fractions, the above expression becomes

$$
\begin{aligned}
F_{2 n 0}= & \frac{1}{\pi}\left(F_{00}-1\right) \frac{1}{\sqrt{3}}(-1)^{n} \frac{\sqrt{2 n}}{2 n}\left[2\left(a_{2 n}\right) O_{0}^{b}-a_{2 n} O_{-2 n}^{b}\right. \\
& \left.-b_{2 n} O_{-2 n}^{a}-a_{2 n} O_{2 n}^{b}+b_{2 n} O_{2 n}^{a}\right]
\end{aligned}
$$

where the quantities appearing in the above expression are defined have been evaluated in [9]. Thus substituting the explicit values of these quantities into (152) and combining terms we find

$$
F_{2 n 0}=\left(F_{00}-1\right) \frac{(-1)^{n} a_{2 n}}{\sqrt{2 n}}
$$

The explicit value of the $F_{00}$ may be computed by substituting (153) into (93). Doing that and rearranging terms we get

$$
\left(F_{00}+1\right)=-2\left(F_{00}-1\right) \sum_{n=1}^{\infty} \frac{a_{2 n}}{2 n}
$$

The sum appearing on the right-hand side has the value $(3 / 2) \ln 3-2 \ln 2$, so we obtain

$$
\frac{1+F_{00}}{1-F_{00}}=\ln \frac{3^{3}}{2^{4}}
$$

which gives the explicit value of $F_{00}$ at once. This result is consistent with that given in [7] [8]. To obtain the explicit value of $F_{02 m}$, we first need to evaluate the sum over $k$ in Equation (94), i.e.,

$$
\sum_{k=1}^{\infty} \frac{(-1)^{k+1}}{\sqrt{2 k}} F_{2 k 2 m}
$$

where the explicit expression for $F_{2 k 2 m}$ in terms of the change of representation matrices is given by Equation (88). Thus substituting (88) into the above expression we have

$$
\begin{aligned}
\sum_{k=1}^{\infty} \frac{(-1)^{k+1}}{\sqrt{2 k}} F_{2 k 2 m}= & \sum_{k=1}^{\infty} \frac{(-1)^{k+1}}{\sqrt{2 k}} \frac{1}{\pi} F_{02 m} \sum_{l=1}^{\infty} \frac{(-1)^{l}}{(2 l-1)^{3 / 2}}\left[\left(M_{1}^{T}+\frac{1}{2} M_{2}^{T}\right)^{-1}\right]_{k l} \\
& -\sum_{k=1}^{\infty} \frac{(-1)^{k+1}}{\sqrt{2 k}} \sum_{l=1}^{\infty}\left[\left(M_{1}^{T}+\frac{1}{2} M_{2}^{T}\right)^{-1}\right]_{k l}\left[\frac{1}{2} M_{1}^{T}+M_{2}^{T}\right]_{l m}
\end{aligned}
$$


If we commute ${ }^{3}$ the sums over $k$ and $l$, we get

$$
\sum_{k=1}^{\infty} \frac{(-1)^{k+1}}{\sqrt{2 k}} F_{2 k 2 m}=\frac{1}{\pi} F_{02 m} \sum_{l=1}^{\infty} \sum_{k=1}^{\infty} \frac{(-1)^{l}}{(2 l-1)^{3 / 2}}(\cdots)-\sum_{l=1}^{\infty} \sum_{k=1}^{\infty}\left[\frac{1}{2} M_{1}^{T}+M_{2}^{T}\right]_{l m}(\cdots)
$$

where

$$
(\cdots) \equiv \frac{(-1)^{k+1}}{\sqrt{2 k}}\left[\left(M_{1}^{T}+\frac{1}{2} M_{2}^{T}\right)^{-1}\right]_{k l}
$$

Substituting Equation (149) for the inverse of the combination $M_{1}^{T}+(1 / 2) M_{2}^{T}$ into the above expression and summing over $k$ from 1 to $\infty$, we obtain

$$
\sum_{k=1}^{\infty} \frac{(-1)^{k+1}}{\sqrt{2 k}}\left[\left(M_{1}^{T}+\frac{1}{2} M_{2}^{T}\right)^{-1}\right]_{k l}=-\frac{2}{\sqrt{3}} \frac{(-1)^{l} a_{2 l-1}}{(2 l-1)^{1 / 2}}
$$

In arriving at the above result we made use of the identities

$$
\sum_{k=0}^{\infty} \frac{a_{2 k}}{2 k-(2 l-1)}=-\frac{1}{2} \sum_{k=0}^{\infty} \frac{a_{2 k}}{2 k+(2 l-1)}=-\frac{1}{2} \frac{1}{\sqrt{3}} \pi a_{2 l-1}
$$

which were derived in [9]. Similar expressions hold for the sums over $b_{2 k}$; see ref. [9]. Now substituting Equation (159) into (157) gives

$$
\sum_{k=1}^{\infty} \frac{(-1)^{k+1}}{\sqrt{2 k}} F_{2 k 2 m}=-\frac{1}{2} \ln \frac{3^{3}}{2^{4}} F_{02 m}+\sum_{l=1}^{\infty} \frac{(-1)^{l}}{\sqrt{3}} \times \frac{2 a_{2 l-1}}{\sqrt{2 l-1}}\left[\frac{1}{2} M_{1}^{T}+M_{2}^{T}\right]_{l m}
$$

Using the explicit value of $M_{1}$ and $M_{2}$ and rewriting $\ln \left(3^{3} / 2^{4}\right)$ in terms of $F_{00}$, the above expression becomes

$$
\sum_{k=1}^{\infty} \frac{(-1)^{k+1}}{\sqrt{2 k}} F_{2 k 2 m}=-\frac{1}{2}\left(\frac{1+F_{00}}{1-F_{00}}\right) F_{02 m}+\frac{(-1)^{m}\left(1-a_{2 m}\right)}{(2 m)^{1 / 2}}
$$

Substituting this result into (94), we find

$$
F_{02 m}=\left(F_{00}-1\right) \frac{(-1)^{m} a_{2 m}}{(2 m)^{1 / 2}}
$$

which has the same form as $F_{2 m 0}$ given in (153). Thus in this case we see that the property $F_{\text {even } 0}=\left(F^{\dagger}\right)_{0 \text { even }}$ holds.

Next we consider the evaluation of $F_{2 n-10}$. If we replace $M_{1}, M_{2}$ and $F_{2 m 0}$ in (92) by their explicit values, given respectively by Equation (66), (67) and (153), we have

$$
\begin{aligned}
F_{2 n-10}= & \frac{2 i}{\sqrt{3}} \frac{2}{\pi}\left(F_{00}-1\right) \frac{(-1)^{n}}{\sqrt{2 n-1}}\left[\frac{1}{2} \sum_{m=1}^{\infty} \frac{a_{2 m}}{2 m-(2 n-1)}\right. \\
& \left.+\sum_{m=1}^{\infty} \frac{a_{2 m}}{2 m+(2 n-1)}\right]+\frac{2 i}{\pi \sqrt{3}} \frac{(-1)^{n}}{(2 n-1)^{3 / 2}}\left(F_{00}-1\right)
\end{aligned}
$$

${ }^{3}$ Since both the sums over $l$ and $k$ are uniformly convergent, one may perform the sums in any order. We have carried the sums in the two different orders and found that the result is the same. However, it is much easier to perform the sum over $k$ first followed by the sum over $l$ rather than the reverse. Here we shall follow the former. 
In order to benefit from the results obtained in [9] to help carry out the sums we first need to extend the range of the sums to include $m=0$. Hence adding zero in the form $-a_{0} /(2 n-1)+a_{0} /(2 n-1)$, the above expression becomes

$$
\begin{aligned}
F_{2 n-10}= & \frac{2 i}{\sqrt{3}} \frac{2}{\pi}\left(F_{00}-1\right) \frac{(-1)^{n}}{\sqrt{2 n-1}}\left[\frac{1}{2} \frac{a_{0}}{(2 n-1)}+\frac{1}{2} \sum_{m=0}^{\infty} \frac{a_{2 m}}{2 m-(2 n-1)}\right. \\
& \left.-\frac{a_{0}}{(2 n-1)}+\sum_{m=0}^{\infty} \frac{a_{2 m}}{2 m+(2 n-1)}+\frac{2 i(-1)^{n}\left(F_{00}-1\right)}{\pi \sqrt{3}(2 n-1)^{3 / 2}}\right]
\end{aligned}
$$

The sums in the square brackets have been evaluated in [9]. Thus one finds

$$
F_{2 n-10}=i\left(F_{00}-1\right) \frac{(-1)^{n} a_{2 n-1}}{\sqrt{2 n-1}},
$$

where $n=1,2,3, \cdots$. To check if the property $F=F^{\dagger}$ continue to hold, we need to compute explicitly the value of $F_{02 n-1}$. It is important to verify that the matrix $F$ is self adjoint for the consistency of our formulation. The matrix element $F_{02 n-1}$ involves the matrix element $F_{2 n 2 k-1}$ which in turn is expressed in terms of the combination $\left(M_{1}^{T}+\frac{1}{2} M_{2}^{T}\right)^{-1}$ and the matrix element $F_{02 n-1}$ itself. To carry out the calculation, unfortunately we first need to compute the explicit value of $F_{2 n 2 k-1}$. The matrix element $F_{2 n 2 k-1}$ is given by (89)

$$
\begin{aligned}
F_{2 n 2 k-1}= & -\frac{i \sqrt{3}}{2}\left[\left(M_{1}^{T}+\frac{1}{2} M_{2}^{T}\right)^{-1}\right]_{n k} \\
& +\frac{1}{\pi} F_{02 k-1} \sum_{m=1}^{\infty}\left[\left(M_{1}^{T}+\frac{1}{2} M_{2}^{T}\right)^{-1}\right]_{n m} \frac{(-1)^{m}}{(2 m-1)^{3 / 2}}
\end{aligned}
$$

Substituting the explicit value of $\left(M_{1}^{T}+\frac{1}{2} M_{2}^{T}\right)^{-1}$ into the above equation and summing over $m$, we find

$$
\begin{aligned}
F_{2 n 2 k-1}= & \frac{(-1)^{n+k} \sqrt{2 n} \sqrt{2 k-1}}{2 i}\left[\frac{a_{2 n} b_{2 k-1}+b_{2 n} a_{2 k-1}}{2 n-(2 k-1)}\right. \\
& \left.+\frac{a_{2 n} b_{2 k-1}-b_{2 n} a_{2 k-1}}{2 n+(2 k-1)}\right]+\frac{(-1)^{n} a_{2 n}}{\sqrt{2 n}} F_{02 k-1}
\end{aligned}
$$

where $n=1,2,3, \cdots$. Combining Equation (168) with Equation (95), leads to

$$
\begin{aligned}
F_{02 m-1}= & i(-1)^{m} \sqrt{2 m-1} \sum_{k=1}^{\infty}\left[\frac{a_{2 k} b_{2 m-1}+b_{2 k} a_{2 m-1}}{2 k-(2 m-1)}\right. \\
& \left.+\frac{a_{2 k} b_{2 m-1}-b_{2 k} a_{2 m-1}}{2 k+(2 m-1)}\right]-2 F_{02 m-1} \frac{1}{2}\left(\frac{1+F_{00}}{1-F_{00}}\right)
\end{aligned}
$$

To evaluate the sums appearing in (169) we first need to extend their range to include $k=0$. Doing so and making use of the result of already established identities in appendix A, Equation (169) reduces to

$$
F_{02 m-1}=2 i(-1)^{m} \frac{a_{2 m-1}}{\sqrt{2 m-1}}-F_{02 m-1}\left(\frac{1+F_{00}}{1-F_{00}}\right)
$$


Solving the above equation for $F_{02 m-1}$, we obtain

$$
F_{02 m-1}=-i\left(F_{00}-1\right)(-1)^{m} \frac{a_{2 m-1}}{\sqrt{2 m-1}}
$$

which is precisely the adjoint of $F_{2 m-10}$; see Equation (166). Thus we have

$$
F_{0 \text { odd }}=\left(F^{\dagger}\right)_{0 \text { odd }}
$$

as expected.

The result obtained in (171) may be now used to find the explicit value of $F_{2 n 2 m-1}$. Thus substituting Equation (171) back into Equation (168), we find

$$
\begin{aligned}
F_{2 n 2 m-1}= & \frac{(-1)^{n+m} \sqrt{2 n} \sqrt{2 m-1}}{2 i}\left[\frac{a_{2 n} b_{2 m-1}+b_{2 n} a_{2 m-1}}{2 n-(2 m-1)}\right. \\
& \left.+\frac{a_{2 n} b_{2 m-1}-b_{2 n} a_{2 m-1}}{2 n+(2 m-1)}\right]-i\left(F_{00}-1\right) \frac{(-1)^{n+m} a_{2 n} a_{2 m-1}}{\sqrt{2 n} \sqrt{2 m-1}}
\end{aligned}
$$

where $n, m=1,2,3, \cdots$.

The computation of the matrix element $F_{2 n-12 m}$ is indeed quite cumbersome. The difficulty arises from the fact that the defining equation of $F_{2 n-12 m}$, which is given by (91), involves this summing over the matrix $F_{2 m 2 k}$ which is potentially divergent when the summing index $m$ takes the $k$ value. The limiting procedures involved in smoothing out the divergence are quite delicate and require careful consideration. Thus here we shall only give the final result; the details may be found in [9],

$$
\begin{aligned}
F_{2 n-12 m}= & -\frac{(-1)^{n+m} \sqrt{2 m} \sqrt{2 n-1}}{2 i}\left[\frac{a_{2 m} b_{2 n-1}+b_{2 m} a_{2 n-1}}{2 m-(2 n-1)}\right. \\
& \left.+\frac{a_{2 m} b_{2 n-1}-b_{2 m} a_{2 n-1}}{2 m+(2 n-1)}\right]+i\left(F_{00}-1\right) \frac{(-1)^{n+m} a_{2 m} a_{2 n-1}}{\sqrt{2 m} \sqrt{2 n-1}}
\end{aligned}
$$

Comparing Equations (173) and (174), we see that

$$
F_{\text {even odd }}=\left(F^{\dagger}\right)_{\text {even odd }}
$$

as expected.

To complete fixing the comma interaction vertex in the full-string basis we still need to compute the remaining elements, namely $F_{2 n 2 m}$ and $F_{2 n-12 m-1}$. The computation of the matrices $F_{2 n 2 m}$ and $F_{2 n-12 m-1}$ involve two distinct cases. The off diagonal case is given by $n \neq m$ and the diagonal case is given by $n=m$. Though the off diagonal elements are not difficult to compute, the diagonal elements are indeed quite involved and they can be evaluated by setting $n=m$ in the defining equations for $F_{2 n 2 m}$ and $F_{2 n-12 m-1}$ and then explicitly performing the sums with the help of the various identities we have established in [9]. An alternative way of computing the diagonal elements is to take the limit of $n \rightarrow m$ in the explicit expressions for the off diagonal elements. We have computed the diagonal elements both ways and obtained the same result which is a non trivial consistency check on our formalism. For illustration, here we shall compute the diagonal elements by the limiting process we spoke of as we 
shall see shortly. But first let us compute the off diagonal elements. We first consider $F_{2 n 2 m}$. From Equation (88), we have

$$
\begin{aligned}
F_{2 n 2 k}= & \frac{1}{\pi} F_{02 k} \sum_{m=1}^{\infty}\left[\left(M_{1}^{T}+\frac{1}{2} M_{2}^{T}\right)^{-1}\right]_{n m} \frac{(-1)^{m}}{(2 m-1)^{3 / 2}} \\
& -\sum_{m=1}^{\infty}\left[\left(M_{1}^{T}+\frac{1}{2} M_{2}^{T}\right)^{-1}\right]_{n m}\left[\frac{1}{2} M_{1}^{T}+M_{2}^{T}\right]_{m k}
\end{aligned}
$$

Substituting the explicit value of $\left(M_{1}^{T}+\frac{1}{2} M_{2}^{T}\right)^{-1}$ and $\frac{1}{2} M_{1}^{T}+M_{2}^{T}$ into the above equation, we have

$$
\begin{aligned}
F_{2 n 2 k}= & F_{02 k} \frac{(-1)^{n} a_{2 n}}{(2 n)^{1 / 2}}-\frac{(-1)^{n+k}(2 k)^{1 / 2}(2 n)^{1 / 2}}{2} \frac{a_{2 n} b_{2 k}+b_{2 n} a_{2 k}}{2 n+2 k} \\
& -\frac{2}{\pi} \frac{(-1)^{n+k}}{\sqrt{3}} \sqrt{2 k} \sqrt{2 n}\left\{\frac{1}{2} \sum_{m=1}^{\infty} \frac{a_{2 n} b_{2 m-1}+b_{2 n} a_{2 m-1}}{[2 n-(2 m-1)][2 k-(2 m-1)]}\right. \\
& \left.+\sum_{m=1}^{\infty} \frac{a_{2 n} b_{2 m-1}-b_{2 n} a_{2 m-1}}{[2 n+(2 m-1)][2 k+(2 m-1)]}\right\}
\end{aligned}
$$

The difficulty in evaluating the sums arises from the fact in performing these sums one usually make use of partial fraction to reduce them to the standard sums treated in [9]; however partial fraction in this case fails due to a divergence arising from the particular case when $n=m$. Thus to carry our program through, we first consider the case for which $n \neq k$. For $n \neq k$, partial fraction can be used to reduce the sums in the above expression to the standard results obtained in [9]. Skipping some rather straight forward algebra, we find

$$
F_{2 n 2 k}=\frac{F_{02 k}(-1)^{n} a_{2 n}}{\sqrt{2 n}}-\frac{(-1)^{n+k} \sqrt{2 k} \sqrt{2 n}}{2}\left[\frac{a_{2 n} b_{2 k}+b_{2 n} a_{2 k}}{2 n+2 k}+\frac{a_{2 n} b_{2 k}-b_{2 n} a_{2 k}}{2 n-2 k}\right]
$$

where $n, k=1,2,3, \cdots$, and $n \neq k$. Substituting the value of $F_{02 k}$, which is given by Equation (163), we have

$$
\begin{aligned}
F_{2 n 2 k}= & \left(F_{00}-1\right) \frac{(-1)^{n+k} a_{2 n} a_{2 k}}{(2 n)^{1 / 2}(2 k)^{1 / 2}}-\frac{(-1)^{n+k}(2 k)^{1 / 2}(2 n)^{1 / 2}}{2} \\
& \times\left[\frac{a_{2 n} b_{2 k}+b_{2 n} a_{2 k}}{2 n+2 k}+\frac{a_{2 n} b_{2 k}-b_{2 n} a_{2 k}}{2 n-2 k}\right]
\end{aligned}
$$

which is the desired result valid for $n, k=1,2,3, \cdots$, and subject to the condition $n \neq k$. Note that in this case we have

$$
F_{\text {even even }}=\left(F^{\dagger}\right)_{\text {even even }}
$$

as expected. As we pointed earlier the diagonal element $F_{2 n 2 k}$ may be obtained by taking the limit of $k \rightarrow n$ in Equation (177). Hence

$$
\begin{aligned}
F_{2 n 2 n}= & F_{02 n} \frac{(-1)^{n} a_{2 n}}{(2 n)^{1 / 2}}-\frac{a_{2 n} b_{2 n}+b_{2 n} a_{2 n}}{4} \\
& -\frac{2}{\pi} \frac{2 n}{\sqrt{3}}\left[\frac{1}{2} a_{2 n} \tilde{S}_{-2 n}^{b}+\frac{1}{2} b_{2 n} \tilde{S}_{-2 n}^{a}+a_{2 n} \tilde{S}_{2 n}^{b}-b_{2 n} \tilde{S}_{2 n}^{a}\right]
\end{aligned}
$$


This result may be simplified further with the help of the following identities derived in [9]

$$
\tilde{S}_{-2 n}^{a}=\frac{1}{2} \tilde{S}_{2 n}^{a}+\frac{1}{4} \pi \sqrt{3} S_{2 n}^{a}, n>0
$$

and

$$
\tilde{S}_{-2 n}^{b}=-\frac{1}{2} \tilde{S}_{2 n}^{b}+\frac{1}{4} \pi \sqrt{3} S_{2 n}^{b}, n>0
$$

Hence

$$
\begin{aligned}
F_{2 n 2 n}= & F_{02 n} \frac{(-1)^{n} a_{2 n}}{(2 n)^{1 / 2}}-\frac{a_{2 n} b_{2 n}+b_{2 n} a_{2 n}}{4} \\
& -\frac{2 n}{\pi} \frac{1}{2}\left[\frac{\pi}{2}\left(a_{2 n} S_{2 n}^{b}+b_{2 n} S_{2 n}^{a}\right)+\sqrt{3}\left(a_{2 n} \tilde{S}_{2 n}^{b}-b_{2 n} \tilde{S}_{2 n}^{a}\right)\right]
\end{aligned}
$$

The generalization of the plus combination in the square bracket has been considered before; its value is given explicitly by setting $p=1 / 3$ in Equation (141)

$$
b_{2 n} S_{2 n}^{a}+a_{2 n} S_{2 n}^{b}=\frac{2}{2 n}
$$

Using this identity, we obtain

$$
F_{2 n 2 n}=F_{02 n} \frac{(-1)^{n} a_{2 n}}{\sqrt{2 n}}-\frac{1}{2} b_{2 n} a_{2 n}-\frac{1}{2}-\frac{2 n}{\pi} \frac{\sqrt{3}}{2}\left(a_{2 n} \tilde{S}_{2 n}^{b}-b_{2 n} \tilde{S}_{2 n}^{a}\right)
$$

Using Equation (163) to eliminate $F_{02 n}$, the above expression becomes

$$
F_{2 n 2 n}=\left(F_{00}-1\right) \frac{a_{2 n} a_{2 n}}{2 n}-\frac{1}{2} b_{2 n} a_{2 n}-\frac{1}{2}-\frac{2 n}{\pi} \frac{\sqrt{3}}{2}\left(a_{2 n} \tilde{S}_{2 n}^{b}-b_{2 n} \tilde{S}_{2 n}^{a}\right)
$$

which satisfies the property

$$
F_{\text {even even }}=\left(F^{\dagger}\right)_{\text {even even }}
$$

as expected.

Finally we consider the matrix elements $F_{\text {odd odd }}$. From Equation (90), we have

$$
F_{2 n-12 k-1}=\frac{2 i}{\sqrt{3}} \sum_{m=1}^{\infty}\left[\frac{1}{2} M_{1}^{T}+M_{2}^{T}\right]_{n m} F_{2 m 2 k-1}+\frac{2 i}{\pi \sqrt{3}} \frac{(-)^{n}}{(2 n-1)^{3 / 2}} F_{02 k-1}
$$

The values of $F_{2 m 2 k-1}$ and $F_{02 k-1}$ are given by Equations (173) and (171) respectively. Hence, substituting the explicit value of $M_{1}^{T}$ and $M_{2}^{T}$ in (189) and skipping some rather straightforward algebra, we find

$$
\begin{aligned}
F_{2 n-12 k-1}= & \frac{(-1)^{n+k}\left(F_{00}-1\right)}{3} \frac{a_{2 k-1} a_{2 n-1}}{(2 n-1)^{1 / 2}(2 k-1)^{1 / 2}} \\
& +\frac{(-1)^{k+n} \sqrt{2 n-1} \sqrt{2 k-1} \frac{a_{2 n-1} b_{2 k-1}+b_{2 n-1} a_{2 k-1}}{(2 n-1)+(2 k-1)}}{2} \\
& +\frac{2(-1)^{k+n}}{\sqrt{3}} \frac{\sqrt{2 k-1} \sqrt{2 n-1} \sum_{m=0}^{\infty}\left[\frac{1}{2} \frac{a_{2 m} b_{2 k-1}+b_{2 m} a_{2 k-1}}{\pi 2 m-(2 n-1)][2 m-(2 k-1)]}\right.}{} \\
& \left.-\frac{a_{2 m} b_{2 k-1}-b_{2 m} a_{2 k-1}}{[2 m+(2 n-1)][2 m+(2 k-1)]}\right]
\end{aligned}
$$


Now there are two cases to consider $k \neq n$ and $k=n$. For $k \neq n$, Equation (190) becomes

$$
\begin{aligned}
F_{2 n-12 k-1}= & \frac{(-1)^{n+k}\left(F_{00}-1\right)}{3} \frac{a_{2 k-1} a_{2 n-1}}{\sqrt{2 n-1} \sqrt{2 k-1}}+\frac{(-1)^{k+n} \sqrt{2 n-1} \sqrt{2 k-1}}{2} \\
& \times\left[\frac{a_{2 n-1} b_{2 k-1}+b_{2 n-1} a_{2 k-1}}{(2 n-1)+(2 k-1)}+\frac{a_{2 n-1} b_{2 k-1}-b_{2 n-1} a_{2 k-1}}{(2 n-1)-(2 k-1)}\right]
\end{aligned}
$$

where $n, k=1,2,3$ and we have made use of the results in [9] to evaluate the various sums. Thus for $n \neq k$, we see that

$$
F_{\text {odd odd }}=\left(F^{\dagger}\right)_{\text {odd odd }} \text {, for } n \neq k
$$

For $k=n$, Equation (190) becomes

$$
\begin{aligned}
F_{2 n-12 n-1}= & \frac{1}{3}\left(F_{00}-1\right) \frac{a_{2 n-1} a_{2 n-1}}{2 n-1}+\frac{1}{2} a_{2 n-1} b_{2 n-1} \\
& +\frac{2}{\sqrt{3}} \frac{(2 n-1)}{\pi}\left\{\frac{b_{2 n-1} \tilde{E}_{-(2 n-1)}^{a}+a_{2 n-1} \tilde{E}_{-(2 n-1)}^{b}}{2}\right. \\
& \left.-\left[b_{2 n-1} \tilde{E}_{(2 n-1)}^{a}-a_{2 n-1} \tilde{E}_{(2 n-1)}^{b}\right]\right\}
\end{aligned}
$$

where we have made use of the results in [9] to evaluate the various sums appearing in the steps leading to the above result. Using the identities

$$
\begin{aligned}
& \tilde{E}_{-(2 n-1)}^{a}=\frac{1}{2} \tilde{E}_{2 n-1}^{a}+\frac{1}{4} \pi \sqrt{3} S_{2 n-1}^{a} \\
& \tilde{E}_{-(2 n-1)}^{b}=-\frac{1}{2} \tilde{E}_{2 n-1}^{b}+\frac{1}{4} \pi \sqrt{3} S_{2 n-1}^{b}
\end{aligned}
$$

derived in [9], the above expression becomes

$$
\begin{aligned}
F_{2 n-12 n-1}= & \frac{1}{3}\left(F_{00}-1\right) \frac{a_{2 n-1} a_{2 n-1}}{2 n-1}+\frac{1}{2} a_{2 n-1} b_{2 n-1}+\frac{1}{2} \\
& +\frac{\sqrt{3}}{2 \pi}(2 n-1)\left(a_{2 n-1} \tilde{E}_{(2 n-1)}^{b}-b_{2 n-1} \tilde{E}_{(2 n-1)}^{a}\right)
\end{aligned}
$$

which is clearly self adjoint. Thus from Equations (191) and (196) it follows that

$$
F_{\text {odd odd }}=\left(F^{\dagger}\right)_{\text {odd odd }}
$$

as expected. With this result we, establish that $F=F^{\dagger}$ as anticipated.

In the original variables, the comma three-string in (60) can be written in the form

$$
\left|V_{x}^{H S}\right\rangle=\delta\left(\sum_{r=1}^{3} p_{0}^{r}\right) V^{H S}\left(\alpha^{1 \dagger}, \alpha^{2 \dagger}, \alpha^{3 \dagger}\right)|0\rangle_{123}
$$

where

$$
V^{H S}\left(\alpha^{1 \dagger}, \alpha^{2 \dagger}, \alpha^{3 \dagger}\right)=\mathrm{e}^{-\frac{1}{2} \sum_{r, s=1}^{3} \sum_{n, m=1}^{\infty} a_{-n}^{r} \mathcal{F}_{n m}^{r s} a_{-m}^{s}}
$$

The matrix elements $\mathcal{F}_{n m}^{i j}$ may be obtained by comparing (199) to (60). For 
example consider the terms involving $a^{1}$ and $a^{2}$ in (60)

$$
\begin{aligned}
- & \frac{1}{2}\left(\frac{1}{3} a_{-n}^{1} C_{n m} a_{-m}^{2}+\frac{1}{3} a_{-n}^{2} C_{n m} a_{-m}^{1}\right)-\left(\frac{1}{3} \bar{e}^{2} a_{-n}^{1} F_{n m} a_{-m}^{2}+\frac{1}{3} e^{2} a_{-n}^{2} F_{n m} a_{-m}^{1}\right) \\
= & -\frac{1}{2} a_{-n}^{1}\left[\frac{1}{3} C-\frac{1}{6}\left(F+F^{T}\right)+i \frac{1}{6} \sqrt{3}\left(F-F^{T}\right)\right]_{n m} a_{-m}^{2} \\
& -\frac{1}{2} a_{-n}^{2}\left[\frac{1}{3} C-\frac{1}{6}\left(F+F^{T}\right)-i \frac{1}{6} \sqrt{3}\left(F-F^{T}\right)\right]_{n m} a_{-m}^{1}
\end{aligned}
$$
obtain

Comparing this result with the terms $-\frac{1}{2} a_{-n}^{1} \mathcal{F}_{n m}^{12} a_{-m}^{2}$ and $-\frac{1}{2} a_{-n}^{2} \mathcal{F}_{n m}^{21} a_{-m}^{1}$, we

$$
\begin{aligned}
\mathcal{F}^{12} & =\frac{1}{6}[(2 C-F-\bar{F})+i \sqrt{3}(F-\bar{F})] \\
\mathcal{F}^{21} & =\frac{1}{6}[(2 C-F-\bar{F})-i \sqrt{3}(F-\bar{F})]
\end{aligned}
$$

where we have used the fact that $F^{T}=\bar{F}$. Likewise one expresses the remaining matrix element $\mathcal{F}_{n m}^{r s}$ in terms of the matrix elements $C_{n m}$ and $F_{n m}$ and their complex conjugates. All in all we have

$$
\begin{aligned}
\mathcal{F}= & \frac{1}{3}\left[(C+F+\bar{F})\left(\begin{array}{ccc}
1 & 0 & 0 \\
0 & 1 & 0 \\
0 & 0 & 1
\end{array}\right)+\left(C-\frac{F+\bar{F}}{2}\right)\left(\begin{array}{lll}
0 & 1 & 1 \\
1 & 0 & 1 \\
1 & 1 & 0
\end{array}\right)\right. \\
& \left.+i \frac{\sqrt{3}}{2}(F-\bar{F})\left(\begin{array}{ccc}
0 & 1 & -1 \\
-1 & 0 & 1 \\
1 & -1 & 0
\end{array}\right)\right]
\end{aligned}
$$

which is the same result obtained in ref. [7]. Equation (203) gives completely the comma interaction three vertex in the full string basis in the representation with oscillator zero modes.

Sometimes it is useful to express the comma vertex in the momentum representation. For a single oscillator with momentum $p$ and creation operator $\alpha^{\dagger}$, the change of basis is accomplished by

$$
\left.p)=\exp \left(-\frac{1}{2} p_{0} \bar{p}_{0}+\bar{p}_{0} \alpha_{0}^{\dagger}+\bar{\alpha}_{0}^{\dagger} p_{0}-\alpha^{\dagger} \bar{\alpha}^{\dagger}\right) \mid 0\right)
$$

with $|0\rangle$ being the oscillator ground state. Thus using the above identity and Equation (61) one finds the following representation for the Vertex in the momentum space

$$
\exp \left[-\frac{1}{2} \sum_{n, m=0}^{\infty} A_{n}^{3 \dagger} C_{n m} A_{m}^{3 \dagger}-\sum_{n, m=1}^{\infty} A_{n}^{\dagger} F_{n m}^{\prime} \bar{A}_{m}^{\dagger}-\sum_{n=0}^{\infty} A_{n}^{\dagger} F_{n 0}^{\prime} \bar{P}_{0}-\sum_{n=0}^{\infty} P_{0} F_{0 m}^{\prime} \bar{A}_{m}^{\dagger}+\frac{1}{2} \bar{P}_{0} F_{00}^{\prime} P_{0}\right]
$$

where the prime matrices $F_{n m}^{\prime}$ are related to the unprimed matrices $F_{n m}$ by

$$
\begin{gathered}
F_{00}^{\prime}=\frac{1+F_{00}}{1-F_{00}} \\
F_{0 n}^{\prime}=\frac{F_{0 n}}{1-F_{00}}, n=1,2,3, \cdots
\end{gathered}
$$




$$
F_{n m}^{\prime}=F_{n m}+\frac{F_{n 0} F_{0 m}}{1-F_{00}}, n, m=1,2,3, \cdots
$$

The property $F^{2}=1$ in Equation (83) implies that in the momentum representation, the $F^{\prime}$ matrix satisfies

$$
\sum_{k=1}^{\infty} F_{n k}^{\prime} F_{k m}^{\prime}=\delta_{n m}, n, m=1,2,3, \cdots
$$

For $n \neq 0$, we have $a_{-n}^{r} \equiv \alpha_{-n}^{r} / \sqrt{n}$, and so Equation (205) may be written as

$$
\begin{aligned}
\left|V_{x}^{H S}\right\rangle= & \int \prod p_{0}^{r} \exp \left[\frac{1}{2} \sum_{r, s=1}^{3} \sum_{n, m=1}^{\infty} \alpha_{-n}^{r} G_{n m}^{r s} \alpha_{-m}^{s}+\sum_{r, s=1}^{3} p_{0}^{r} G_{0 m}^{r s} \alpha_{-m}^{s}\right. \\
& \left.\left.+\frac{1}{2} \sum_{r, s=1}^{3} p^{r} G_{00}^{r s} p_{0}^{s}\right] \mid 0, p\right)_{123}
\end{aligned}
$$

where the matrix $G$ is defined through the relation

$$
G_{n m}^{r s}=-\frac{1}{\sqrt{n+\delta_{n 0}}} \mathcal{F}_{n m}^{\prime r s} \frac{1}{\sqrt{m+\delta_{m 0}}}
$$

The ghost part of the comma vertex in the full string basis has the same structure as the coordinate one apart from the mid-point insertions

$$
\left|V_{\phi}^{H S}\right\rangle=\mathrm{e}^{\frac{1}{2} i \sum_{r=1}^{3} \phi^{r}(\pi / 2)} V_{\phi}^{H S}\left(\alpha^{\phi, 1 \dagger}, \alpha^{\phi, 2 \dagger}, \alpha^{\phi, 3 \dagger}\right) 0, N_{\text {ghost }}=\frac{3}{2}>_{123}^{\phi}
$$

where the $\alpha^{\prime}$ s are the bosonic oscillators defined by the expansion of the bosonized ghost $\left(\phi(\sigma), p^{\phi}(\sigma)\right)$ fields and $V_{\phi}^{H S}\left(\alpha^{\phi, 1 \dagger}, \alpha^{\phi, 2 \dagger}, \alpha^{\phi, 3 \dagger}\right)$ is the exponential of the quadratic form in the ghost creation operators with the same structure as the coordinate piece of the vertex.

\section{Conclusion}

We have successfully constructed the comma three interaction vertex of the open bosonic string in terms of the oscillator representation of the full open bosonic string. The form of the vertex we have obtained for both the matter and ghost sectors are those obtained in ref. [7] [8] [10]. This establishes the equivalence between Witten's 3-interaction vertex of open bosonic strings and the half string 3-vertex directly without the need for the coherent state methods employed in ref. [1].

\section{Conflicts of Interest}

The authors declare no conflicts of interest regarding the publication of this paper.

\section{References}

[1] Bordes, J., Hong-Mo, C., Nellen, L. and Sheung, T. (1991) Nuclear Physics B, 351, 441-473. https://doi.org/10.1016/0550-3213(91)90097-H

[2] Abdurrahman, A., Anton, F. and Bordes, J. (1993) Nuclear Physics B, 397, 260-282. https://doi.org/10.1016/0550-3213(93)90344-O 
[3] Abdurrahman, A, Anton, F. and Bordes, J. (1994) Nuclear Physics B, 411, 694-714. https://doi.org/10.1016/0550-3213(94)90467-7

[4] Abdurrahman, A. and Bordes, J. (2001) Nuovo Cimento B, 116, 635.

[5] Abdurrahman, A. and Bordes, J. (2003) Nuovo Cimento B, 118, 641.

[6] Abdurrahman, A. and Bordes, J. (2003) Nuovo Cimento B, 116, 635-658.

[7] Gross, D.J. and Jevicki, A. (1987) Nuclear Physics B, 283, 1-49. https://doi.org/10.1016/0550-3213(87)90260-4

[8] Gross, D.J. and Jevicki, A. (1987) Nuclear Physics B, 287, 225-250. https://doi.org/10.1016/0550-3213(87)90104-0

[9] Gassem, M. (2008) The Operator Connecting the SCSV 3-Vertex and the Comma 3-Vertex. PhD Thesis, The American University, London. https://doi.org/10.1103/PhysRevD.49.2966

[10] Itoh, K., Ogawa, K. and Suchiro, K. (1987) Nuclear Physics B, 289, 127. https://doi.org/10.1016/0550-3213(87)90374-9 Siegel, W. (1984) Physics Letters B, 149, 157-161. https://doi.org/10.1016/0370-2693(84)91574-0

Hata, H., Itoh, K., Kugo, T., Kunimoto, H. and Ogawa, K. (1986) Physics Letters B, 172, 186. https://doi.org/10.1016/0370-2693(86)90834-8

Neveu, A. and West, P. (1986) Physics Letters B, 165, 63-66.

https://doi.org/10.1016/0370-2693(85)90691-4 Friedan, D. (1985) Physics Letters B, 162, 102-108.

Gervais, J.L. (1986) l'Ecole Normale Superieure Preprints LPTENS, 85/35. https://doi.org/10.1016/0370-2693(85)91069-X Gervais, J.L. (1986) l'Ecole Normale Superieure Preprints LPTENS 86/1. Neveu, A. and West, P. (1986) Physics Letters B, 168, 192. https://doi.org/10.1016/0370-2693(86)90962-7 Chang, N.P., Guo, H.Y., Qiu, Z. and Wu, K. (1986) City College Preprint. Tseytlin, A.A. (1986) Physics Letters B, 168, 63. https://doi.org/10.1016/0370-2693(86)91461-9 